\title{
RELACIONES INTERSECTORIALES EN MAGALLANES: EN BUSCA DE SU ESTRUCTURA ECONÓMICA
}

SERGIO SOZA AMIGO*

Recibido: 01/01/2008

Aceptado: 23/05/2008

\begin{abstract}
RESUMEN
El establecer cuáles son las ramas claves de una economía, siempre ha sido un tema recurrente, en especial, bajo un entorno de análisis input-output. Esto sería causado por dos razones: la primera, tendría que ver con el liderazgo que puede tener una actividad sobre el resto, que se entiende es motivado por el posible impulso que puede dar un sector al desarrollo económico de una determinada zona; $y$, por otra parte, debido a que el concepto de rama "clave", ha sido presa de controversias, dado que es muy amplio e impreciso, ya que el mismo depende de la perspectiva en que se aborde el problema en cuestión.

En este trabajo, se realiza un estudio que permite jerarquizar, desde múltiples ángulos, la importancia de las actividades económicas de la Región de Magallanes y la Antártica Chilena. Para ello, se hace uso de las tablas input-output desarrollada por el INE-Chile y MIDEPLAN, cuyos datos son referidos a 1996. De esta forma, primero se presenta un análisis de encadenamientos, donde se emplean para su determinación las técnicas de Rasmussen (1956), Chenery y Watanabe (1958), Hazari (1970), Cella (1985), Sonis et al (1995), Dietzenbacher y van der Linden (1997) y Soza (2007), cuyas respuestas finalmente se conjugan en la obtención de unos indicadores sintéticos, que facilitan su interpretación y evitan, de igual manera, una profusión de resultados. En segundo lugar, se determina la sensibilidad de los coeficientes técnicos, empleando la propuesta de Schintke y Stäglin (1988), proceso que ha permitido identificar la importancia de una rama, en función de su elasticidad, por el número de coeficientes más sensibles que presenta en columnas como en filas, y, en función de la alteración de cada uno de los coeficientes por separado, siguiendo el planteamiento de Aroche, en el sentido de utilizar la teoría de Grafos (Aroche, 1996; 2002 y 2005), se han determinado los campos de influencia de cada actividad.

Una vez realizado todo lo anterior, y en base a los principales resultados obtenidos, se procedió a la identificación de aquellas ramas que son motivadoras de desarrollo para la región materia de estudio.
\end{abstract}

PALABRAS CLAVES: Análisis Estructural, Sectores Claves, Encadenamientos, Análisis de Sensibilidad, Coeficientes Importantes, Campos de Influencia.

* Profesor Asociado, Universidad de Magallanes, Facultad de Ciencias Económicas y Jurídicas. Avenida Bulnes 01855, Punta Arenas, e-mail: sergio.soza@umag.c

Investigación financiada por CONICYT-FONDECYT, caratulado como proyecto número 11070168. 


\title{
INTERSECTORIAL RELATIONS IN MAGALLANES IN SEARCH OF ITS ECONOMIC STRUCTURE
}

\begin{abstract}
It has always been a recurring theme to be able to establish what the key areas of an economy are, especially under an environment of input-output analysis. This would be caused by two reasons, the first would be related to the leadership that one particular activity can have on the other, what is meant to be motivated by the possible impulse that a particular sector can give to the economic development of a particular area, and on the other side, due to the fact that the concept of branch "key" has been subject to disputes for being very broad and vague, since it depends on the perspective that addresses the issue at hand.

This study allows for a multiple-angle hierarchically of the importance of economic activities in the Region of Magallanes and the Chilean Antarctic. In order to do that, input-output tables developed by the INE-Chile and MIDEPLAN are used, and which data refer to 1996. Firstly, a linkage analysis is presented, and various techniques are used for identification [Rasmussen (1956), Chenery and Watanabe (1958), Hazari (1970), Cella (1985), Sonis et al (1995), Dietzenbacher and van der Linden (1997) and Soza (2007)]. The answers are finally combined to obtain some synthetic indicators, which facilitate their interpretation as well as avoiding a profusion of results. Secondly, the sensible of technical coefficients is determined using the Schintke and Stäglin proposal (1988), which has identified the importance of a branch in terms of its elasticity, given the number of more sensible coefficients presented in columns as well as rows, and depending on the alteration of each of the separate factors and following Aroche's approach, in the sense of using Graph Theory (1996; 2002 and 2005), influence fields for have been identified each activity

After carrying out all of the above, and based on the main results achieved, the following step was the identification of those groups which generate evolvement in the region studied.
\end{abstract}

KEY WORDS: Structural Analysis, Key Sector, Linkage, Sensible Analysis, Important Coefficients, Influence Fields.

\section{INTRODUCCIÓN}

Sobre la base de las consecuencias que puede originar el contar con un estudio para la economía magallánica, en donde se identifiquen las interrelaciones de una actividad económica, se ejecuta el siguiente trabajo, cuyo objetivo prioritario es la individualización de ramas claves, desde la óptica de las características recientemente mencionadas; esto es, por un lado, se indaga qué sectores inducen al desarrollo de otras ramas por la vía de las compras y ventas de insumos, $y$, por otro, se observa, desde la perspectiva de las ramas que albergan un mayor número de coeficientes sensibles, cuál de ellas es más importante para el desarrollo de otras, pero en un sentido de dependencia para las demás. En otras palabras, la primera parte de este trabajo, se centra en observar cómo una rama "tira" y/o "empuja" del resto del sistema económico; y en la segunda, se presta atención a la importancia que tiene, por un lado, el método de producción que en ella se emplea, $y$, por otro, las implicancias que tiene el desarrollo tecnológico de una rama para el resto de las actividades. Es decir, se presenta un proceso complementario al anterior, en donde se evalúa cómo la posible modificación de una etapa, o bien, la función productiva de una rama, o el conjunto de funciones productivas del sistema, afectan a la economía.

Para cumplir con el cometido trazado, se utiliza el entorno input-output. Entre las ventajas de este planteamiento, se puede señalar la forma en que se limita el marco de trabajo, quedando circunscrito a la propia economía; en concreto, a la tabla input-output que se emplee. Dicha limitante, permite identificar cuáles son las interdependencias que existen entre los distintos bienes y servicios que han sido elaborados, y consumidos por el propio sistema productivo que se analiza.

Las tablas input- output (TIO) o matrices de insumo- producto (MIP), son de doble entrada y describen los flujos económicos de una determinada 
área. Desde esta perspectiva, una MIP se centra en las ramas de actividad, las que están compuestas por unidades de producción homogéneas, unidades institucionales y finalmente unidades de actividad económica local.

Bajo un punto de vista contable, una TIO señala en forma sistemática, las consecuencias propias de una recopilación de datos estadísticos. Por lo tanto, gracias a ella se pueden observar las transacciones que se realizan en una economía, entre una y otra rama, más los inputs primarios o factores primarios y la demanda final. Por tanto, bajo esta condición, ofrece un ángulo cuantitativo de la información recogida $y$, por ende, del entramado económico- contable imperante.

Una TIO dada la información que incorpora, señala la interdependencia productiva de las distintas actividades. Bajo este prisma, muestra que sectores son claves, no sólo desde un punto de vista cuantitativo, sino que también e inevitablemente, desde la función que ellas ejercen en la transmisión de los impulsos que brindan a la propia estructura económica. En este sentido, ambos enfoques son vitales para el planificador, ya que él debe centrar la mirada no sólo en actividades de gran envergadura, sino que también, en aquellas que ofrecen más flujo de intercambio de bienes y servicios.

En resumen, una TIO muestra el funcionamiento básico de una determinada estructura productiva, es decir, su entramado o esqueleto que lo sostiene, permitiendo por tanto, dar una importancia distinta a las relaciones de intercambio. En otras palabras, a través de ella se puede separar lo realmente importante o significativo de lo poco relevante. Esto es, facilita el trabajo y las conclusiones derivadas del estudio abordado, al aislar las relaciones significativas o de mediana importancia, propias de la estructura económica analizada, de las que son débiles, despreciables o simplemente independientes.

Esquemáticamente, una TIO está compuesta por tres submatrices: la matriz de transacciones intermedias, la de inputs primarios y recursos, y la de empleos finales. La matriz de transacciones intermedias muestra los consumos que se generan a través del proceso productivo entre los distintos bienes y servicios, en términos de recursos y empleos, es decir, como entradas y salidas de una rama. Según se desee aplicar un enfoque de oferta o de demanda, se analizará por filas o columnas. Si se realiza un análisis por filas, digamos sobre la rama i-ésima, cada una de ellas indicará el consumo que todos los sectores efectúan de esta. Por lo que se refiere a las columnas, cada una representa los bienes y servicios provenientes del resto de la economía y en algunos casos del propio sector, cuyo objetivo es permitir en este caso, que la rama i-ésima, pueda lograr su producción.

La submatriz de inputs primarios y recursos, mostrará los componentes que constituyen el valor añadido (VA) de la rama correspondiente, si además se incorporan la suma de los consumos intermedios (CI) y el excedente de explotación (EE), mostrará el input total (IT).

Los empleos finales, están formada por demanda final (DF) requerida de cada rama, la cual está compuesta por el consumo privado (C) y, colectivo o gasto público (G), formación bruta de capital (I) $y$, exportaciones (E). Por otro lado, si al conjunto de los consumos intermedios en filas se les suma su correspondiente demanda final, se obtiene el vector columna del output total (OT), que es igual al vector fila de input total (IT).

El estudio del tipo de relaciones interindustriales que posee una actividad económica (en un entorno input-output), se suele abordar desde dos perspectivas: una, en la cual se identifica la importancia de ella por medio de sus encadenamientos (linkage), y otra, en donde se presta atención al impacto que tiene, sobre el resto del sistema económico, una pequeña alteración generada ex profeso en una etapa (un coeficiente) o en el conjunto de las etapas productivas de las distintas ramas de actividad (análisis de sensibilidad). Tratar estos aspectos es un asunto recurrente, cuando está de por medio la realización de estudios que permiten la caracterización de sectores. Lo anterior, obedece a que cada rama genera distintos efectos sobre su entorno, en función de las capacidades que tiene para inducir el desarrollo de otras actividades económicas, ya sea, por la vía de la compra de insumos (productos semi elaborados o finales), o bien, por la venta de los mismos. De esta forma, dichos enfoques (linkage y análisis de sensibilidad), permiten obtener una visión más significativa, en un sentido pormenorizado, del entramado económico que se analiza.

En lo referente al ejercicio que se aplica a la región materia de estudio, él se realiza puesto 
que nos ha parecido interesante indagar por su estructura económica desde distintas perspectivas, esto es, comprender su funcionamiento por la vía de sus encadenamientos, coeficientes más sensibles $y$, sobre la base de los campos de influencia que se forman. Conocer estas características, tiene la ventaja de permitir comprender los posibles efectos de determinadas políticas económicas, además se facilita el entendimiento de la forma en que la región se ha venido desarrollando. Aunque claro ésta, aún cuando se repliquen ciertas medidas que en antaño tuvieron cierto éxito -ya sean a nivel local o en otras regiones-, ellas aseguren que el mismo se repita.

Para lograr lo trazado, y en los términos aquí establecidos, se necesita utilizar una tabla inputoutput. En este sentido, se debe tener claro cuál es la data de la tabla a utilizar, su origen, de que tipo es y su tamaño. Ella fue elaborada con datos de 1996, es confeccionada por MIDEPLAN ${ }^{1}$, es simétrica ${ }^{2}$ y se ha creído oportuno trabajar con su nivel de desagregación original, es decir, de 40 ramas $^{3}$. Si bien es cierto que, emplear una tabla tan antigua, puede considerarse que va en desmedros de las conclusiones, ello obedece a que no existen tablas más actualizadas a nivel regional. Por otra parte, aunque se podría haber actualizado los datos mediante técnicas indirectas, e.g. la RAS, ello hubiera significado, por una parte, emplear como base la tabla de 1996, y, por otra, hubiese implicado reducir el número de sectores a por lo menos la mitad, ya que los datos requeridos, para tal ejercicio, no existen para todas las ramas. Por ello, se creyó más significativo utilizar la información proporcionada por MIDEPLAN.

1 MIDEPLAN. Aproximación a las Economías Regionales con base en las Matrices de Insumo Producto Regionales del Año 1996. Santiago, Chile, Ministerio de Planificación, 2005, pp. 211.

2 En estricto rigor, ella se debiera expresar cómo producto por producto, a pesar de ello, en este documento se trata como rama por rama y, aún cuando el concepto de rama es distinto al de sector, estos términos aquí se emplean como sinónimos.

3 En principio se considero más oportuno trabajar con 64 ramas, esto es, con la Matriz Insumo Producto Regional de 1996, elaborada por el Instituto Nacional de Estadísticas (Santiago, Chile, 2004), sin embargo, esto se desestimo debido a la forma en que venía confeccionada tal tabla. Sin perjuicio de ello, también se analizo y proceso dicha matriz, cuyos principales resultados sirvieron posteriormente para validar los obtenidos con la matriz finalmente empleada.
En otro orden de cosas, trabajar con tal tamaño (40 sectores), si bien es cierto significa una mayor dificultad, tiene la ventaja que, por su nivel de detalle, permitirá ahondar e identificar claramente aquellos sectores en los cuales se debiera indagar aún más el análisis estructural. Por otro lado, adicionalmente, tiene la particularidad de facilitar el análisis concerniente a la importancia individual de cada coeficiente; esto es, la identificación de aquellas etapas productivas que fueron más influyentes en el entramado económico de la región magallánica.

\section{INTERRELACIONES SECTORIALES}

El hecho que las tablas input-output se presenten como matrices de doble entrada, permite la identificación de la importancia y magnitud que tienen los inputs intermedios, tanto para las actividades que los demandan como para las que los ofrecen, facilitando de esta forma la tipificación de ramas de actividad "clave", en función de las interrelaciones productivas que ella posea; esto es, se logra tipificar la relevancia de una actividad de acuerdo a la magnitud de sus relaciones, ya sea por la vía de la demanda (modelo de Leontief), o de la oferta (modelo de Ghosh). Dado que se ha propuesto, como objetivo prioritario en este documento, la identificación de ramas "claves", esto es, el plasmar desde distintas perspectivas cuál o cuáles de las actividades que se realizan en la región magallánica destacan por ser vitales o impulsoras para el desarrollo económico de la región, lo que sigue guarda relación precisamente con tal proceso, el cual se aborda básicamente desde cuatro ángulos. El primero, vincula la magnitud e importancia que tiene una rama sobre el total, esto es, se centra en la participación que tienen las distintas ramas en los consumos intermedios, valor añadido y producción, sobre los correspondientes totales. En segundo lugar, se trata el objetivo propuesto desde una arista en donde se consideran tanto los efectos directos como indirectos; bajo este prisma, la selección de un sector relevante se hará en función del valor que éste ocupe respecto a una media global (encadenamientos). En lo referente a los dos últimos procedimientos, ellos se basan en el análisis de sensibilidad, el que básicamente permite identificar la importancia de una actividad económica, en función del máximo porcentaje de variación por el cual puede verse afectada una actividad, sin 
Tabla 1: Importancia \% de cada sector según su participación en $\mathrm{CI}^{4}, \mathrm{VA}^{5}$, producción (X) e Importaciones

\begin{tabular}{|c|c|c|c|c|c|}
\hline \multicolumn{2}{|c|}{ Ramas } & \multirow{3}{*}{\begin{tabular}{r|} 
Producción \\
$\mathrm{X}$ \\
6.69
\end{tabular}} & \multicolumn{2}{|c|}{ Doméstico } & \multirow{2}{*}{$\begin{array}{c}\text { Importaciones } \\
\text { CI }\end{array}$} \\
\hline & & & \multirow{2}{*}{\begin{tabular}{|l|} 
CI \\
7.60 \\
\end{tabular}} & \multirow{2}{*}{\begin{tabular}{|l|}
$\mathrm{VA}$ \\
3.89
\end{tabular}} & \\
\hline 3 & Ganado; productos pecuarios y carne & & & & 11.91 \\
\hline 5 & Pescados y Mariscos & 7.76 & 8.71 & 7.87 & 6.20 \\
\hline 6 & Petróleo crudo y combustible y lubricantes & 15.58 & 22.45 & 11.42 & 15.75 \\
\hline 19 & Productos químicos, de acucho y plástico & 6.18 & 6.68 & 8.35 & 0.49 \\
\hline 27 & Construcción & 6.52 & 4.66 & 6.56 & 8.98 \\
\hline 28 & Servicios comerciales & 13.12 & 11.82 & 9.77 & 22.62 \\
\hline 29 & Servicios hotel y restaurantes & 5.54 & 6.09 & 5.29 & 5.38 \\
\hline 30 & Transporte ferroviario y caminero & 3.90 & 3.72 & 4.49 & 2.79 \\
\hline 35 & Servicios empresariales & 4.32 & 3.42 & 4.91 & 4.19 \\
\hline 36 & Propiedad de vivienda & 5.22 & 4.29 & 7.93 & 0.23 \\
\hline & Resto & 25.17 & 20.56 & 29.52 & 20.28 \\
\hline & Total en porcentaje (\%) & 100,00 & 100,00 & 100,00 & 100,00 \\
\hline
\end{tabular}

Fuente: Propia, en base a MIDEPLAN 2005.

que ello signifique una alteración en la producción; de esta forma, se analiza, por un lado, cuál es la elasticidad de las distintas etapas productivas, y por otro, cuáles son sus campos de influencia.

\section{IMPORTANCIA DE UNA RAMA EN FUNCIÓN DE SU PARTICIPACIÓN}

En lo relativo a la importancia de los sectores (tabla 1), se abordó este punto considerando el peso que posee cada uno en el valor de su producción (X), consumos intermedios (CI) nacionales e importados, y valor añadido (VA). Sobre la base de la información anterior, se analizó cuales son las actividades que más destacan, desde dos perspectivas: una, considerando el entorno doméstico (flujos interiores), y, en el otro, el exterior (flujos de importación).

De acuerdo a la tabla 1, se aprecia cual es la importancia relativa del 25\% de los sectores con mayor producción durante 1996, y cuáles son las distintas participaciones de estas actividades en los consumos intermedios, valores añadidos e importaciones, sin incluir, de momento, la importancia de las relaciones interindustriales, las cuales pueden arrojar conclusiones distintas a las obtenidas siguiendo este procedimiento.

Como se puede ver, bajo el esquema recientemente indicado, las actividades más importantes en Magallanes están vinculadas a la extracción y refinación de petróleo, y a los servicios comerciales.

\footnotetext{
Consumo Intermedio.

Valor Añadido.
}

Por otra parte, se observa que actividades tales como la agricultura, pesca, construcción, y servicios de hotel y restaurantes, tienen una importancia no menor en el desarrollo económico de esta región.

En otro orden de cosas, también llama la atención, la concentración de esta producción en la región, prueba de ello es que el $25 \%$ de las actividades más importantes, concentran casi el 75\% de ella; situación similar ocurriría con los consumos intermedio, valor añadido e importaciones.

Otro punto que merece ser comentado, tiene que ver con las participaciones no homogéneas que presenta el sector servicios comerciales, que, por un lado, sobresale en referencia a los demás, por lo alto de su participación en las importaciones, y por otro, destaca el mismo por lo bajo de sus consumos intermedios y valor añadido, siendo esto un claro reflejo del escaso aporte directo de este sector a la región, ello en un sentido de desarrollo económico. Sin embargo, se entiende que el destino de sus productos cumple otro rol, el cual estaría asociado a actividades más bien de tipo final, tales cómo, consumo de hogares.

\section{EFECTOS DIRECTOS E INDIRECTOS}

Los efectos directos e indirectos de una economía, bajo el entorno input-output, se obtienen a partir de matrices inversas. En este sentido, se distinguen dos enfoques para su interpretación: uno de demanda, y otro de oferta. En el caso del primero, su matriz inversa depende de la matriz 
de coeficientes técnicos $\mathrm{A}$, la que es resultado de dividir cada inputs por su output total en un sentido vertical, obteniéndose con ello las distintas funciones de producción de cada actividad. Desde el segundo punto de vista, dicha inversa se basa en la matriz de coeficientes de distribución $\mathrm{B}$, la cual es obtenida de dividir cada inputs por su output total, pero a diferencia del anterior, esta vez en un sentido horizontal; luego, cada fila de la matriz B, representará la función de distribución de la rama analizada. De este modo, ambos enfoques en conjunto permiten identificar la importancia de una actividad, de acuerdo a dos criterios, de demanda para el primero, y de oferta para el segundo.

Una de las ventajas de trabajar con el modelo de demanda, es que la matriz inversa de Leontief ${ }^{6}$, muestra cuál es el grado de interdependencia de los elementos propios del sistema. Es decir, cada coeficiente de dicha matriz indica en cuánto debe variar éste, a fin de satisfacer la variación de la demanda final de cada uno de los demás sectores.

Por su parte, la interpretación del enfoque de oferta, que se basa en la matriz inversa de Ghosh ${ }^{7}$, tiene un significado similar a la anterior, ella señala en cuánto debe variar cada coeficiente, a fin de satisfacer la variación de los input primarios de cada una de las demás ramas.

Desde las perspectivas anteriores, se hace atractivo el análisis estructural en un contexto inputoutput, ya que permite la individualización de las actividades económicas que inducen al desarrollo. El planteamiento como tal, se acuño a partir de la noción de eslabonamiento propuesta por Hirschman (1958, pp. 110), que nace en respuesta a los tipos de interdependencia que existen entre los sectores de una economía. En este sentido, establece que un sector presenta fuertes encadenamientos hacia atrás (backward linkages; BL) si demanda gran cantidad de inputs del resto, de manera que induce el desarrollo de otras actividades; por otra parte, un sector presenta fuertes encadenamientos hacia delante (forward linkages; FL), si del desarrollo de

6 El cual se expresa como: $\mathbf{x}=(\mathbf{I}-\mathbf{A})^{-1} \mathbf{y}$; donde $\mathbf{x}$ representa la producción, I es una matriz diagonal unitaria e, y la demanda final, la cual incluye el consumo privado, público, la formación de capital y exportaciones.

7 La que matricialmente se representa de la siguiente manera: $\mathbf{x}^{\mathbf{t}}=\mathbf{v}(\mathbf{I}-\mathbf{B})^{-\mathbf{1}}$; donde el superíndice $\mathbf{t}$, indica que es un vector transpuesto, $\mathbf{v}$ representa a los input primarios (salarios, excedente bruto de explotación, valor añadido).
Cuadro 1. Clasificación de los sectores según los eslabonamientos.

\begin{tabular}{|l|l|l|}
\hline & BL <Promedio (BL) & BL >Promedio (BL) \\
\hline $\begin{array}{l}\text { FL }<\text { Promedio } \\
(\text { FL) }\end{array}$ & $\begin{array}{l}\text { Sectores } \\
\text { independientes }\end{array}$ & $\begin{array}{l}\text { Sectores } \\
\text { impulsores }\end{array}$ \\
\hline FL $>$ Promedio (FL) & Sectores base & Sectores claves \\
\hline
\end{tabular}

Fuente: Propia en base a Rasmussen (1956) y Hirschman (1958).

su actividad se logran productos que utilizarán otras ramas para su proceso productivo. A partir de estos últimos conceptos se puede efectuar la clasificación que se muestra en el cuadro 1.

Con el fin de comprender la importancia que tiene una rama y el origen de su clasificación, a continuación se pasa a detallar cuál es su naturaleza, esto es, su génesis desde la perspectiva de Hirschman. Serán sectores base, los que presenten una baja demanda de inputs, en cambio muestran una alta oferta de los mismos al sistema. En este sentido, su producción se destina preferentemente a un uso intermedio; es decir, son inputs que sirven a otros sectores. Los sectores impulsores, o de arrastre de una economía, son grandes demandantes de insumos intermedios y, dada la capacidad que tienen de inducir otras actividades, pueden afectar en mayor cuantía al crecimiento global de la misma; sin embargo, son ramas que poco ofrecen, pero si demandan más en términos relativos que el resto. Los sectores independientes son aquellos que presentan unos eslabonamientos hacia delante y hacia atrás por debajo de la media, no afectando por tanto, al desarrollo de otras actividades. Por último, los sectores claves, demandan y ofrecen en términos relativos grandes cantidades de inputs intermedios, luego son una parte importante del traspaso de actividades de toda economía (ver cuadro 1).

Como se puede ver, la identificación de la importancia de las distintas características de una rama según Hirschman, servirá de guía para sentar distintas políticas con la finalidad de obtener un pronto desarrollo económico, ya que éste, estaría fuertemente relacionado con las ramas en las cuales se invierta. Si se parte del supuesto caeteris páribus, y se potencian aquellos sectores que están muy interrelacionados con el resto, esto es, tanto por la vía de la demanda como por el de la oferta, con mayor prontitud se alcanzará el desarrollo, en caso contrario, más lento será ello, ya que sus efectos no 
repercuten sobre la totalidad o mayoría del sistema productivo (Hirschman, 1958, pp. 106).

En la actualidad, existe un nutrido grupo de técnicas (clásicas, extracción hipotética y de descomposición) que permiten determinar los eslabonamientos hacia delante y hacia atrás en una economía, entre los cuales podemos mencionar los más utilizados, a saber, dentro de las técnicas clásicas, estarían las propuestas de Rasmussen (1956), Chenery-Watanabe (1958) y Hazari (1970); por su parte, las de extracción hipotética más conocidas y utilizadas en la actualidad son: las de Strassert (1968), Cella (1984) y Dietzenbacher y van der Linden (1997); y, finalmente, en las de descomposición, la de Sonis et al (1995) ${ }^{8}$, agregando en esta línea la reciente propuesta de Soza en 2007.

A continuación, y con el fin de cumplir el cometido impuesto, esto es, identificar las distintas tipologías de las actividades económicas para la Región de Magallanes y la Antártica Chilena, se utilizarán para tal efecto una propuesta clásica (Rasmussen), una de extracción hipotética (Dietzenbacher y van der Linden), y una de las modificaciones que plantea Soza en 2007, y que se enmarca dentro del enfoque de descomposición ${ }^{9}$. Con el fin de facilitar tal proceso, la idea central de cada una de estas alternativas de evaluación, se explican en los párrafos siguientes.

\section{PODER Y SENSIBILIDAD DE DISPERSIÓN}

Rasmussen, en 1956, a partir del promedio de la suma de las columnas y filas de la matriz inversa de Leontief, presentó los hoy en día conocidos índices de poder y sensibilidad de dispersión. Para

8 Para un mayor detalle de estas técnicas puede revisar SOZA, Sergio. Análisis de la Economía Chilena a Partir de una Matriz Insumo-Producto, Economía y Administración, Universidad de Concepción, Concepción, Chile, 41(63): 63-86, 2004. O bien: SOZA, Sergio. Análisis Estructural Input-Output: Antiguos problemas y nuevas soluciones. Tesis (Doctorado en Economía Aplicada). Oviedo, España, Universidad de Oviedo, Departamento de Economía Aplicada, 280 p., 2007.

9 Aún cuando, se analizó desde otras perspectivas, los distintos valores que pueden tomar los encadenamientos (CheneryWatanabe (1958), Hazari (1970), Cella (1980) y Sonis et al (1995), sus principales resultados no se incluyen en las primeras etapas de este trabajo, ello con el fin de evitar una profusión de información que, más que esclarecer las conclusiones, las nublan; sin embargo, ellos fueron considerados al momento de tipificar cada actividad en una etapa posterior de este documento. el primero, señala que representa, en términos relativos, los efectos directos $e$ indirectos que se dispersan por todo el sistema económico, como consecuencia de los cambios que se generan sobre la producción total, cuando ella es influenciada por el aumento de una unidad de la demanda final de la j-ésima rama, es decir, el índice señala cómo un aumento de la producción, de la j-ésima actividad, "empuja" al sistema económico (ecuación 1).

$$
\mathbf{B L}^{\mathrm{R}}=\frac{\mathbf{n i}^{\mathrm{t}}(\mathbf{I}-\mathbf{A})^{-1}}{\mathbf{i}^{\mathrm{t}}(\mathbf{I}-\mathbf{A})^{-1} \mathbf{i}}
$$

Por su parte, el índice de sensibilidad de dispersión (ecuación 2), muestra cómo un aumento de la demanda final del conjunto de actividades económicas, "tira" de la rama i-ésima. Es decir, señala cómo el sector i-ésimo es afectado por una expansión del sistema de industria, o bien, en palabras de Rasmussen, cómo el aumento de demanda de una industria (sector) elegida al azar, afecta a la producción de la i-ésima rama (Rasmussen, 1956, pp. 129).

$$
\mathbf{F L}^{\mathrm{R}}=\frac{\mathbf{n}(\mathbf{I}-\mathbf{A})^{-1} \mathbf{i}}{\mathbf{i}^{\mathrm{t}}(\mathbf{I}-\mathbf{A})^{-1} \mathbf{i}}
$$

Sobre la base de lo que calcula el índice anterior, y debido a su ambigüedad, ciertos autores, entre los que se encuentran Augustinovics (1970, pp. 252-253), Jones (1976, pp. 326-327 y 329), Beyers (1976, pp. 231), Miller y Lahr (2000, pp. 3), Andreosso-O Callaghan (2000, pp. 5 y 2004, pp. 168) y Soza (2007, pp. 20-22), sugieren la necesidad de emplear el modelo de Ghosh para determinar los FL en lugar del modelo de Leontief. En concreto, Jones (1976, pp. 326-327 y 329) señala que los indices son insensibles ya que no capturan la realidad de la economía, pues cree más adecuado relacionar al FL con un modelo de oferta y no con uno que se derive de la demanda. En esta misma línea también se puede señalar los trabajos de Beyers (1976), Miller y Lahr (2000) y Andreosso-O Callaghan (2000 y 2004), los cuales indican que el FL de Rasmussen cuantifica el aumento de una unidad en la demanda final de todos los sectores, lo que según Miller y Lahr 
(2000) es poco claro y habría originado el uso de la matriz de distribución de Ghosh.

Luego, emplear el modelo de Ghosh depende de qué es lo que mide, y puesto que cuantifica la proporción de las ventas que realiza la i-ésima rama al resto de la economía, se acepta que es una medida acertada para la interpretación de los multiplicadores de oferta. Concluyendo, la expresión del FL de Rasmussen, en términos de la matriz de Ghosh, sería:

$$
F L^{R-G}=\frac{n(I-B)^{-1} i^{t}}{i(I-B)^{-1} i^{t}}
$$

De esta forma, ahora el índice de sensibilidad de dispersión (ecuación 3), cuantifica, en términos relativos, cuál es la proporción de las ventas que realiza la i-ésima rama al resto de la economía o, en términos más precisos, identifica cuál es la cuantía en que aumenta la producción, cuando se incrementan en una unidad los inputs primarios de la i-ésima rama.

\section{EXTRACCIÓN HIPOTÉTICA}

La idea de extraer una rama de la economía, surge en 1965 con el trabajo desarrollado por de Caevel, Degueldre y Paelinck, planteamiento que fue continuado por Ronald Miller en 1966. Sin embargo, fue Günter Strassert, en 1968, el que continuó y ahondó en el tema, y al que se le atribuye esta propuesta metodológica. Este autor retoma la idea de extracción, mostrando el interés de cuantificar cómo se ve afectada la economía cuando se retira de ella una determinada rama. Destaca la importancia de evaluar las repercusiones que se pueden obtener en los encadenamientos cuando se eliminan algunos elementos.

Strassert plantea este enfoque como una alternativa de evaluación respecto a los métodos clásicos. Este autor, propone, a partir de la inversa de Leontief, cuantificar el efecto que se produciría en una economía, si se extrajera hipotéticamente una rama, para lo cual, sugiere eliminar la fila (ventas), columna (compras) y demanda final (y) de la i-ésima rama, logrando extraer literalmente el sector, estableciendo de esta forma el modelo que se muestra en la ecuación (4).

$$
\mathbf{L}(\mathbf{k})=\sum_{\mathrm{i}-1,1=\mathbf{k}}^{\mathrm{n}}\left[\mathbf{x}_{\mathrm{i}}-\overline{\mathbf{x}}_{\mathrm{i}}(\mathbf{k})\right]
$$

Donde $\mathrm{L}(\mathrm{k})$ es el eslabonamiento total del sector, $\mathrm{x}_{\mathrm{i}} \overline{\mathrm{yx}}_{\mathrm{i}}(\mathrm{k})$, representan su output antes y después de la extracción respectivamente, por su parte $\mathrm{x}$ se puede obtener de la siguiente forma:

$$
\mathrm{x}=(\mathrm{I}-\mathrm{A})^{-1} \mathrm{y}
$$

Análogamente, la producción $\overline{\mathrm{x}}(\mathrm{k})$ de la ecuación (4) se puede reescribir como:

$$
\overline{\mathbf{x}}(\mathbf{k})=[\mathbf{I}-\overline{\mathbf{A}}(\mathbf{k})]^{1} \overline{\mathbf{y}}(\mathbf{k})
$$

Donde $[\mathrm{I} \overline{\mathrm{A}}(\mathrm{k})]^{-1}$, es la matriz inversa de Leontief, de orden [(n-1)*(n-1)], ya que a la matriz de coeficientes técnicos se le ha eliminado la fila $y$ columna del sector $k$-ésimo, por su parte, $\bar{x}(\mathrm{k}) e$ $\bar{y}(\mathrm{k})$, representarán los nuevos vectores de output y demanda, ambos de dimensión [(n-1)*1].

Posteriormente en 1997, Dietzenbacher y van der Linden, retoman la idea anterior, pero agregando a tal planteamiento, las ideas expresadas en los trabajos de Augustinovics (1970), Beyers (1976), Jones (1976), Meller y Marfán (1981) y Cella (1984). Plantean que la forma más adecuada de determinar los eslabonamientos es, partiendo de la metodología de Cella, emplear el modelo de Leontief (de demanda) para el cálculo de los BL y el de Ghosh (de oferta) para la determinación de los FL.

De acuerdo a lo anterior, para el cálculo del $\mathrm{BL}_{\mathrm{j}}^{\mathrm{D}-\mathrm{vdL}}$, se toma la matriz columna $\overline{\mathrm{x}}_{\mathrm{i}}(\mathrm{k})$, y se asume que el sector a evaluar, no se interrelaciona con el medio por la vía de las compras (en este caso el uno y en adelante el j-ésimo), es decir, no compra insumos derivados de su producción para satisfacer su demanda $\left(A_{11}=0\right)$, ni los que provéniesen de otros sectores $\left(A_{21}=0\right.$; ecuación 7$)$.

$$
\overline{\mathbf{x}}_{j}(\mathbf{k})=\left[\begin{array}{l}
\overline{\mathbf{x}}_{1} \\
\overline{\mathbf{x}}_{22}
\end{array}\right]=\left[\begin{array}{ll}
0 & \mathbf{A}_{12} \\
0 & \mathbf{A}_{22}
\end{array}\right]\left[\begin{array}{l}
\overline{\mathbf{x}}_{1} \\
\overline{\mathbf{x}}_{2}
\end{array}\right]+\left[\begin{array}{l}
\mathbf{y}_{1} \\
\mathbf{y}_{2}
\end{array}\right]
$$


Porlotanto, el $\mathbf{B L}_{\mathbf{j}}^{\mathrm{D}-\mathbf{v d L}}=\left[\mathbf{i}^{\mathbf{t}}\left(\mathbf{x}-\overline{\mathbf{x}}_{\mathbf{j}}(\mathbf{k})\right) / \mathbf{x}_{\mathbf{j}}\right] \mathbf{1 0 0}$ indicará, para la condición establecida, cómo se ve afectada la economía, cuando su demanda final aumenta en una unidad monetaria.

A su vez, cuando establecen el FL $\mathrm{F}_{i}^{\mathrm{D}-\mathrm{dd}}$, parten del mismo supuesto, lo cual da lugar a que la fila de la rama que se analiza tome el valor cero en la matriz de distribución, es decir, tal rama no vende insumos, esto es, se anula su autoconsumo $\left(\mathrm{B}_{11}=0\right)$, y las ventas que esta realiza resto de la economía $\left(\mathrm{B}_{12}=0\right.$; ecuación (8).

$$
\overline{\mathbf{x}}^{\mathrm{t}}=\left[\begin{array}{ll}
\overline{\mathbf{x}}_{1}^{\mathrm{t}} & \overline{\mathbf{x}}_{2}^{\mathrm{t}}
\end{array}\right]=\left[\begin{array}{ll}
\overline{\mathbf{x}}_{1}^{\mathrm{t}} & \overline{\mathbf{x}}_{2}^{\mathrm{t}}
\end{array}\right]\left[\begin{array}{cc}
\mathbf{0} & \mathbf{0} \\
\mathbf{B}_{21} & \mathbf{B}_{22}
\end{array}\right]+\left[\begin{array}{ll}
\mathbf{v}_{1} & \mathbf{v}_{2}
\end{array}\right]
$$

Por lo tanto, el $\mathbf{F L}_{\mathbf{i}}^{\mathbf{D}-\mathbf{v d L}}=\left[\left(\mathbf{x}^{\mathbf{t}}-\overline{\mathbf{x}}_{\mathbf{i}}^{\mathbf{t}}(\mathbf{k})\right) \mathbf{i} / \mathbf{x}_{\mathbf{i}}\right] \mathbf{1 0 0}$ , donde $\overline{\mathbf{x}}^{\mathbf{t}}(\mathbf{k})$ es el transpuesto del output total de la rama i-ésima, representará el impacto que se produce en la economía, cuando se extrae la i-ésima rama, indicando de esta forma, cómo se ve afectada la producción cuando sus inputs primarios aumentan en una unidad monetaria.

\section{REPLANTEAMIENTO DEL ENFOQUE DE DESCOMPOSICIÓN}

En lo relativo al origen de este enfoque, él nace de la propuesta de Sonis et al (1995), el que básicamente consiste en descomponer la economía, y no en extraer una parte de ella. Aún que, se considere que la idea intuitiva que existe tras la descomposición propuesta por Sonis et al es correcta, por incluir en ella, por ejemplo, la relación que existe entre la rama que se evalúa con el resto de la economía y su producción, se cree que es susceptible de mejoras, ya que no capta adecuadamente la concentración de compras y ventas, y no emplea un enfoque de oferta para el cálculo del encadenamiento hacia delante (FL). Respecto a este último punto, aún cuando Andreosso-O'Callaghan y Yue (2000, pp. 10 y 2004, pp. 170), proponen una alternativa más correcta que la original para determinar el FL, en el sentido de considerar como más idóneo el empleo de la matriz inversa de Ghosh, no se está de acuerdo en la forma en que ella se aplica, ya que la propuesta de AndreossoO'Callaghan y Yue se aleja de la definición original de Sonis et al. Se consideró que un planteamiento más coherente, consiste en seguir el procedimiento de dichos autores. Por lo tanto, si se quiere corregir esta propuesta mediante el empleo de la matriz de distribución, la matriz $\mathrm{B}$ se debiera descomponer en:

$$
\mathbf{B}=\left[\begin{array}{ll}
\mathbf{B}_{11} & \mathbf{B}_{12} \\
\mathbf{B}_{21} & \mathbf{B}_{22}
\end{array}\right]=\left[\begin{array}{cc}
\mathbf{B}_{11} & \mathbf{B}_{12} \\
\mathbf{B}_{21} & \mathbf{0}
\end{array}\right]+\left[\begin{array}{cc}
\mathbf{0} & \mathbf{0} \\
\mathbf{0} & \mathbf{B}_{22}
\end{array}\right]=\mathbf{B}_{1}+\mathbf{B}_{2}
$$

Donde $\mathrm{B}_{11}$ se asocia a su autoconsumo; $\mathrm{B}_{12}$ a las ventas que este realiza al resto del sistema económico; $\mathrm{B}_{21}$ a sus compras, $\mathrm{y}$, finalmente, $\mathrm{B}_{22}$, representa al resto de la economía.

Luego, y sobre la base de la descomposición anterior, se obtiene la inversa de Ghosh; posteriormente, esta se descompone, con fin de analizar los elementos que determinan conceptualmente el FL, de esta forma se plantea que, el PFL ${ }_{\mathrm{i}}^{\mathrm{G}}=\mathrm{v}_{1} \mathrm{~B}_{12} \mathrm{~W}_{22} \mathrm{i}^{\mathrm{t}}$, donde $\mathrm{W}_{22}=\left(\mathrm{I}-\mathrm{B}_{22}\right)^{-1}$, refleja cómo la i-ésima rama afecta a la producción del resto de los sectores cuando su input primario aumenta en una unidad.

Por otra parte, si bien es cierto que, la idea de Sonis et al está bien concebida, se observa que, en el cálculo de los eslabonamientos originales, se presenta alguna carencia desde el punto de vista de lo que se evalúa. En esta línea, y en contra de los que sostienen Cai y Leung (2005, pp. 49), cuando afirman que emplear en tales índices la producción, está bien, estimo que es más importante, debido a su significación, evaluar en los distintos encadenamientos las repercusiones que tiene la demanda final (para el caso del BL), y los inputs primarios (para los FL), y no cuantificar las repercusiones que tiene la modificación de la producción total de la rama que se estudia en el resto del sistema, o bien, evaluar cómo afecta la producción del resto de la economía a la rama que se analiza.

Lo anterior, obedece a que la idea central de tal enfoque es determinar los "puros encadenamientos" o las puras relaciones que se asocian al impacto que tiene la rama en el entorno, sin considerar sus propios consumos; esto es, lo que se estudia con tal propuesta es, cómo afecta la modificación de la producción de la rama que se analiza al resto de la economía, sin incluir su autoconsumo. Sin embargo, si se utiliza la producción, en ella va implícita parte de su autoconsumo, por tanto, se es de la opinión que es mejor analizar 
para tales efectos, cuál es la repercusión que tiene la modificación de una unidad de su demanda final (o input primarios) en el resto de la economía, y no la de su producción, ya que finalmente, en general, lo que interesa pesquisar son los impactos que tiene la rama sobre su entorno, y no sus efectos sobre sí misma.

Por último, y considerando que, para el cálculo del BL, es más adecuado emplear una formulación que recoja, por un lado, el sentido económico de lo que se quiere indagar, y por otro, presente un respaldo matemático, se cree que es más acertado emplear la expresión (10):

$$
\mathrm{PBL}_{\mathrm{j}}^{\mathrm{c}}=\mathrm{i}^{\mathrm{t}} \mathrm{G}_{22} \mathrm{~A}_{21} \mathrm{y}_{1}
$$

Donde $G_{22}=\left(I-A_{22}\right)^{-1}$, representa el efecto multiplicador de lo que queda de la economía $\left(\mathrm{A}_{22}\right)$, y $\mathrm{A}_{21}$ corresponde a las compras que realiza, en este caso, la rama 1, al resto del sistema económico; por tanto, la ecuación 10, señalará cómo afecta la variación de la demanda de la rama que se estudia al resto del sistema económico.

Sin embargo, y al igual que en otros casos, esta propuesta puede estar sesgada, al no considerarse aspectos tales como, la ponderación ${ }^{10}$, y la dispersión ${ }^{11}$, de los efectos generados en la economía ${ }^{12}$.

La forma en que se propone solucionar los aspectos recientemente mencionados es, empleando el ponderador de Rao y Harmston (1979, pp. 83), esto es, un coeficiente será clave cuando tenga tanto su FL como BL una vez relativizados sobre la unidad y además esté ponderado (ecuación 11 y

10 Si ella no se incluye, se puede erróneamente identificar actividades importantes, por el tamaño que poseen, y no por el tipo de relaciones que generan; un ejemplo de tal situación son las administraciones públicas.

11 Este aspecto tiene que ver con el grado de concentración del efecto, es decir, se evalúa, si un determinado impacto, afecta a pocas ramas o, a muchas, a modo de muestra, se pueden considerar los impactos que generaría en Chile, un cambio en la demanda del cobre (poca dispersión, o pocas actividades afectadas), y otra, relacionada con la alteración de la demanda del sector agrícola (mucha dispersión).

12 Aún cuando se sugiere considerar la ponderación y dispersión de las distintas actividades, para el caso concreto de esta propuesta ello pasa a depender de la economía que se estudia y del criterio por el que opte el analista, pues en algunos casos en los cuales está todo muy bien distribuido, ello no es estrictamente necesario, por otro lado, no se trabaja con la inversa de Leontief ni con la de Ghosh en su conjunto, sino que con una parte de ella, lo que permitiría aislar ciertos efectos.
14), y posea coeficientes de variación de pequeña cuantía (ecuación 13 y 16), es decir:

$$
\mathrm{PFL}_{\mathrm{i}}^{\mathrm{G}, \mathrm{w}}=\mathrm{PFL}_{\mathrm{i}}^{\mathrm{G}} \mathrm{W}_{\mathrm{i}}
$$

\section{Donde}

$\mathbf{w}_{i}=\left\{\begin{array}{l}\frac{\mathbf{x}_{\mathbf{i}}}{\mathbf{x}}, \text { si i es un sector de un pais desarollado } \\ \frac{\mathbf{y}_{\mathbf{i}}}{\mathbf{y}}, \text { si i es un sector en un país en vías de desarrolla }\end{array}\right.$

$$
\text { Luego, } \quad v_{i}^{*}=\frac{\sqrt{\frac{1}{n-1} \sum_{i=1}^{n}\left(g_{v}-\frac{x_{i}}{x} g_{t}\right)^{2}}}{\frac{x_{i}}{x} g_{t}}
$$

Donde

$$
\mathbf{v}_{\mathrm{i}}^{\mathrm{w}}=\frac{\sqrt{\frac{1}{\mathbf{n}-1} \sum_{\mathrm{j}-1}^{\mathrm{n}}\left(\mathrm{g}_{\mathrm{ij}}-\frac{\mathbf{X}_{\mathrm{i}}}{\mathbf{x}} \mathbf{g}_{\mathrm{i}}\right)^{2}}}{\frac{\mathbf{X}_{\mathrm{j}}}{\mathbf{x}} \mathbf{g}_{\mathrm{i}}}
$$

$\mathrm{i}=1,2, \ldots, \mathrm{n}, \mathrm{y}$ además $\mathrm{g}_{\mathrm{ij}} \in \mathrm{G}=(\mathrm{I}-\mathrm{B})^{-1}$.

$Y$ para el caso del encadenamiento hacia atrás $\mathrm{PBL}_{\mathrm{j}}^{\mathrm{c}, \mathrm{w}}=\mathrm{PBL}_{\mathrm{j}}^{\mathrm{c}} \mathrm{W}_{\mathrm{j}}$

$\mathbf{w}_{\mathbf{j}}=\left\{\begin{array}{l}\text { Donde } \\ \frac{\mathbf{X}_{\mathbf{j}}}{\mathbf{x}}, \text { si i es un sector de un país desarollado } \\ \frac{\mathbf{y}_{\mathbf{i}}}{\mathbf{y}}, \text { si i es un sector en un pais en vías de desarrollo }\end{array}\right.$

Finalmente,

$$
\mathbf{P B L}_{\mathrm{j}}^{\mathrm{c}-\mathrm{p}}=\mathbf{P B L}_{\mathrm{j}}^{\mathrm{c}, \mathrm{w}}\left(\frac{\mathbf{v}_{\mathrm{j}}^{\mathrm{w( \text {(niv) }}}}{\operatorname{máx}\left(\mathbf{v}_{\mathrm{j}}^{\mathbf{w ( n i v )}}\right)}\right)
$$


Tabla 2: Encadenamientos hacia atrás según distintas técnicas.

\begin{tabular}{|c|c|c|}
\hline $\begin{array}{c}\text { Rasmussen } \\
(1956)\end{array}$ & $\begin{array}{c}\text { Dietzenbacher- van der Linden } \\
(1997)\end{array}$ & $\begin{array}{c}\text { Soza } \\
(2007)\end{array}$ \\
\hline Ramas > a la media: 21 & Ramas > a la media: 21 & Ramas > a la media: 10 \\
\hline \multicolumn{2}{|c|}{10 ramas con mayores efecto según las distintas técnicas utilizadas } \\
\hline productos agrícolas & productos agrícolas & pesca \\
\hline Frutas & frutas & petróleo (combustible y lubricante) \\
\hline ganado; productos pecuarios y carne & petróleo (combustible y lubricante) & otros minerales \\
\hline Pesca & otros minerales & química, petróleo, cuacho y plástico \\
\hline petróleo (combustible y lubricante) & aceite y grasas & construcción \\
\hline aceite y grasas & química, petróleo, cuacho y plástico & servicios comerciales \\
\hline química, petróleo, cuacho y plástico & vidrio y minerales no metálicos & servicios de hotel y restaurantes \\
\hline vidrio y minerales no metálicos & resto industria manufacturera & propiedad de vivienda \\
\hline resto industria manufacturera & gas y agua & administración pública \\
\hline gas y agua & servicios de hotel y restaurantes & salud pública y privada \\
\hline
\end{tabular}

Fuente: Propia.

$v_{i}^{w}=\frac{\sqrt{\frac{1}{n-1} \sum_{i-1}^{n}\left(z_{i j}-\frac{X_{j}}{x} z_{j}\right)^{2}}}{\frac{x_{j}}{x} z_{j}} \quad, \operatorname{con} j=1,2, \ldots$, $n y, z_{i j} \in Z=(I-A)^{-1}$.

Como se puede ver, este planteamiento tiene, entre otras ventajas, las de, por un lado, corregir desde una óptica de oferta el cálculo del FL. Al proponer utilizar la inversa de Ghosh, con ello se logra determinar cuál es la cuantía necesaria en que se deben acrecentar los inputs primarios de una rama en concreto, a fin de conseguir un aumento unitario en el sector que se estudia, por otra parte, se estaría incluyendo el efecto exógeno que genera la rama que se estudia en la economía. Además, se excluye del indicador los efectos endógenos al considerar sólo los exógenos, esto es, se relaciona en el caso del BL, el efecto que tiene un cambio unitario de la rama que se estudia en las compras que ésta realiza al resto del sistema económico, las que, a su vez, se relacionan con lo que queda de la economía; en otras palabras, se evalúa cómo el cambio de la demanda de un sector (en este caso el del uno; $y_{1}$ ), afecta a, las compras que realizan de él, el resto del sistema económico $\left(A_{21}\right)$, excluyendo en este caso las propias $\left(\mathrm{A}_{11}\right) \mathrm{y}$, además, se cuantifica cómo estas compras $\left(\mathrm{A}_{21}\right)$, afectan al resto del sistema económico $\left(\mathrm{G}_{22}\right)$. Situación similar ocurriría con el FL, ya que ahora se relaciona el efecto

Tabla 3: Encadenamientos hacia delante según distintas técnicas.

\begin{tabular}{|c|c|c|}
\hline $\begin{array}{c}\text { Rasmussen (Ghosh) } \\
\text { (1956) }\end{array}$ & $\begin{array}{c}\text { Dietzenbacher- van der Linden } \\
\text { (1997) }\end{array}$ & $\begin{array}{l}\text { Soza } \\
(2007)\end{array}$ \\
\hline Ramas > a la media: 18 & Ramas > a la media: 14 & Ramas > a la media: 10 \\
\hline \multicolumn{3}{|c|}{10 ramas con mayores efecto según las distintas técnicas utilizadas } \\
\hline productos agrícolas & productos agrícolas & petróleo (combustible y lubricante) \\
\hline petróleo (combustible y lubricante) & impresos y grabaciones & química, petróleo, cuacho y plástico \\
\hline impresos y grabaciones & vidrio y minerales no metálicos & electricidad \\
\hline vidrio y minerales no metálicos & hierro, acero y productos metálicos & construcción \\
\hline hierro, acero y productos metálicos & electricidad & servicios comerciales \\
\hline electricidad & transporte ferroviario y caminero & servicios de hotel y restaurantes \\
\hline transporte ferroviario y caminero & transporte marítimo y aéreo & transporte ferroviario y caminero \\
\hline transporte marítimo y aéreo & comunicaciones & servicios financieros y seguros \\
\hline servicios empresariales & servicios empresariales & servicios empresariales \\
\hline resto servicios personales & resto servicios personales & propiedad de vivienda \\
\hline
\end{tabular}

Fuente: Propia. 
de un cambio unitario en los inputs primarios de una rama, en este caso la uno $\left(\mathrm{v}_{1}\right)$, con su oferta al resto del sistema productivo $\left(\mathrm{B}_{12}\right) \mathrm{y}$, se observa cómo esta afecta al resto del sistema económico $\left(\mathrm{W}_{22}\right)$.

\section{IDENTIFICACIÓN DE SECTORES CLAVES PARA LA ECONOMÍA MAGALLÁNICA}

A continuación, se pasa a mostrar sobre la base de las técnicas presentadas en los párrafos precedentes, cuáles son los encadenamientos más importantes para la región materia de estudio. Ello se realizó sólo centrándose en las actividades vinculadas a la producción doméstica, y en aquellas que presentaron un encadenamiento superior a la media de la técnica empleada. Esto obedece a dos razones, la primera tiene que ver con el hecho de que es la estructura económica propia de la región la que interesa, esto es, la importancia que tiene cada rama, y sus interrelaciones sectoriales a nivel local, ya que las mismas son derivadas de la producción nacional, y en segundo lugar, porque es allí donde podrían tener una mayor injerencia las conclusiones que emanan de este trabajo.

De acuerdo a los resultados obtenidos, y que se muestran en las tablas 2 y 3 , ellas señalan, por un lado, las 10 actividades más relevantes respecto a su media, $y$, además, indican cuál es el número de sectores que están sobre tal valor que, en general, para las tres técnicas, los resultados son para las ramas más importantes, similares, por tanto, se facilita la identificación de ramas claves.

Otras actividades tales cómo; cobre, conservas de frutas y vegetales, industria de la leche, molinería y panaderías, elaboración de alimentos para animales, producción de bebidas alcohólicas, analcohólicas y vinos, elaboración de productos del tabaco, textil, prendas vestir y cuero, maquinarias y equipos eléctricos, y servicios vinculados a la educación pública y privada, resultaron ser islas o independientes, es decir, su desarrollo no afectó al resto, pues su vínculo con otras actividades, ya sea por la vía de la oferta o demanda, fue escaso.

En función de lo expuesto, y de los resultados obtenidos en base a las distintas técnicas, serían ramas motivadoras de desarrollo para la región materia de estudio -recordando que se trabaja bajo el supuesto caeteris paribus-, las actividades vinculadas a la pesca, extracción y refinación de petróleo, parte de la minería (donde se excluye extracción y refinación de petróleo, y cobre), química, petróleo, caucho y plástico, construcción, transporte en general, servicios comerciales, servicios de hotel y restaurantes, y comunicaciones.

Por otra parte, también se aprecian los sectores que eran impulsoras de la actividad económica durante 1996, al interior de la región materia de estudio, siendo éstos, precisamente, aquellos ligados a servicios, actividades propias de una economía desarrollada. Además se observa, en base a las tablas 2 y 3 , qué ramas eran base, esto es, aquellas cuya actividad se relaciona más con la oferta que con la demanda de inputs; por ejemplo, los servicios financieros y compañías de seguros, y servicios empresariales.

\section{INTERRELACIONES ECONÓMICAS: UNA VISIÓN GLOBAL PARA LAS DISTINTAS TÉCNICAS QUE DETERMINAN ENCADENAMIENTOS}

En lo que continua, se analiza la economía magallánica desde una perspectiva global; en este sentido, lo que sigue tiene por finalidad proporcionar una alternativa de análisis tal, que facilite el estudio de la estructura de una economía en un entorno input-output, pero esta vez, en forma amplia, facilitándose con ello la comprensión de lo estudiado, evitando de esta forma, las consecuencias propias que trae consigo una gran profusión de resultados. El objetivo que se persigue, por tanto, es caracterizar y clasificar un sector de acuerdo a los valores de sus encadenamientos en forma conjunta, ya que, si bien los distintos enfoques que permiten realizar dicho análisis (clásicos, extracción hipotética o descomposición) ${ }^{13}$, determinan el tipo de influencia que ejerce cada rama en la economía, no siempre enfocan ni evalúan de la misma manera el problema; es decir, aunque presentan ciertas similitudes, no existe un planteamiento ni patrón de respuestas análogas. Además, trabajar con una batería de indicadores dificulta el análisis, sobre todo si el estudio se hace empleando un gran número de metodologías y ramas. Se considera que una alternativa que permite resolver éste problema es empleando indicadores

13 Se excluyen de este análisis de momento los coeficientes importantes, ya que lo que se pretende encontrar es un $\mathbf{B L}$ y FL que resuma el conjunto de indicadores empleados. 
sintéticos, los cuales pueden ser obtenidos de las Técnicas de Análisis Multivariante (Soza y Ramos (2005, pp. 371) y, Soza (2007, pp. 169)).

Por tanto, lo que continua está centrado en la obtención de dos indicadores sintéticos: uno, relativo al eslabonamiento hacia delante (BL), y, otro, hacia atrás (FL), que faciliten el análisis de la estructura productiva de la región materia de estudio. Esto es, teniendo como base un conjunto de encadenamientos medidos según distintas metodologías, se procede a realizar un análisis estructural tomando el conjunto de los procedimientos anteriormente mencionados [Rasmussen (1956), Chenery y Watanabe (1958), Hazari (1970), Cella (1984), Sonis et al (1995), Dietzenbacher y van der Linden (1997), y la alternativa recientemente presentada por Soza en 2007]. De esta manera, se espera lograr superar el inconveniente de analizar múltiples procedimientos, ya que de acuerdo a los resultados empíricos, se observa que, entre las distintas propuestas, no existe un patrón común en cuanto a las respuestas proporcionadas por cada una, aún cuando sí se observan ciertas coincidencias. La forma de proceder ha sido aplicando la técnica de Análisis Factorial, esto es, se han buscado dos indicadores sintéticos, uno para la determinación del BL y otro para la del FL, separando de esta forma los encadenamientos hacia atrás de los hacia adelante. El propósito de ello, es la obtención de una clasificación similar a la presentada anteriormente (sectores claves, impulsoras, base o islas), pero, para esta ocasión, con una mirada de conjunto y no de casos particulares.

En el análisis realizado, no se ha considerado la propuesta de Cella debido a una decisión de carácter puramente conceptual, ya que dicho enfoque no es directamente comparable con el resto. Sin embargo, también es cierto que, si se hubiesen tenido en cuenta los resultados empíricos emanados de esta técnica, no habrían empeorado las conclusiones, sino por el contrario, ya que, en general, se facilitaba la clasificación (situación similar ocurriría con el planteamiento de Hazari $\left.^{14}\right)$. Por último, se debe señalar que los FL utilizados están corregidos mediante el empleo de la matriz de Ghosh, es decir, se considera para su determinación un enfoque de oferta.

A continuación, se verificará si los datos a emplear se adecuan a los supuestos de la técnica

14 En anexo 2 se incluyen los resultados obtenidos a partir de ambas técnicas. que se utilizará. Para ello se analizará la matriz de correlaciones, el test de Bartlett, la prueba KMO y el análisis de la matriz de correlación anti-imagen.

En base a la matriz de correlaciones, $y$ al valor de su determinante, se observa que, en general, los valores están sobre el umbral generalmente utilizado (30\%; Hair et al, 1999, pp. 88); además, su determinante presentó en ambos casos, un valor cercano a cero. Sobre ésta base, se puede afirmar que los datos se adecuan a la técnica, ya que este último valor, señala que el grado de ausencia de correlación de los datos es muy alto, condición que se requiere para emplear la técnica de componentes principales.

Con el fin de contrastar formalmente la existencia de correlación, se utiliza el test de esfericidad de Bartlett, el cual señala que existe una correlación significativa entre las variables, ya que su valor resultó ser nulo tanto para las variables que se vinculan al BL como al FL.

Para evaluar si el análisis está apropiadamente realizado, se empleó el estadístico $\mathrm{KMO}$, en ambos casos, esto es, para los BL y FL, y se observó que tal estadístico está por sobre 0.5 (0.591 y 0.857 , respectivamente), confirmándose, por tanto, que el análisis esta adecuadamente aplicado.

De la matriz de correlaciones anti-imagen, se observa que, en general, todos los valores son bajos, por tanto, se constata nuevamente la idoneidad de la técnica a los datos utilizados.

Comprobado lo anterior, lo siguiente será obtener los distintos indicadores sintéticos, esto es, uno para el BL y otro para el FL.

De acuerdo a lo ejecutado, se puede señalar que los factores retenidos representan adecuadamente a las variables originales, y responden a los límites aceptados en ciencias sociales (alrededor del 60\%; Hair et al, 1999, pp. 92).

En lo referente a las comunalidades, se observa que son todas próximas a 0.90 , por tanto, cada una de las variables iníciales está adecuadamente representada por los factores retenidos (Cuadro 2).

Finalmente, se analizó la bondad del modelo a partir de la matriz de correlaciones reproducida, y en ambos casos se constató que, el número de residuos no redundantes, con valores absolutos mayores que 0.05 , es bajo, 2 para el caso del BL, que representan el 20\%, y ninguno, para el caso del FL (0\%). Es decir, las diferencias encontradas son aceptables (Pérez, 2005, pp. 511). 
Cuadro 2: Proporción de inercia retenida por los factores (indicador sintético del BL y FL).

\begin{tabular}{|l|c|c|c|}
\hline Encadenamiento & $\begin{array}{c}\text { Proporción } \\
\text { de inercia } \\
\text { retenida, } 1^{\circ} \\
\text { factor }\end{array}$ & $\begin{array}{c}\text { Proporción } \\
\text { de inercia } \\
\text { retenida, } 1^{\circ} \\
\text { factor }\end{array}$ & $\begin{array}{c}\text { Proporción } \\
\text { acumulada } \\
\text { de inercia } \\
\text { retenida }\end{array}$ \\
\hline BL & 77.057 & 18.390 & 95.446 \\
\hline FL & 79.059 & 17.525 & 96.585 \\
\hline
\end{tabular}

Fuente: Propia.

Posteriormente, se rotó la matriz de componentes mediante el método VARIMAX, y se observó que en ambas componentes, y para los dos casos, las correlaciones son altas y positivas.

Una vez realizado lo anterior, el último paso consiste en rescatar la información sintetizada. Para ello se usa la matriz de coeficientes para el cálculo de las puntuaciones en los componentes, y dado que se han retenido dos factores, los indicadores son calculados como una media ponderada por las varianzas explicadas. Se ha optado por este criterio, ya que según Castro (2002, pp. 272), se atiende tanto a las cargas que son mayores como a las menores, obteniéndose, por tanto, mejores conclusiones.

Gráfico 1: Clasificación de ramas de acuerdo a los indicadores sintéticos obtenidos ${ }^{15}$.

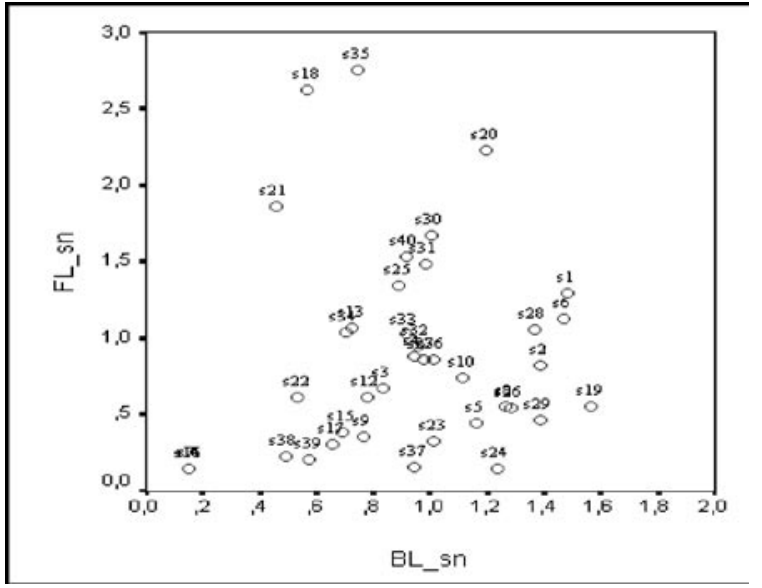

Fuente: Propia.

Con el fin de observar los resultados obtenidos, esto es, el BL y FL sintetizado de la región materia

15 En anexo 2, en columna "factorial", se muestra en forma más detalla la información presentada en el gráfico 1 . de estudio, se construyo el gráfico 1con los mismos. El gráfico resultante, al cual le fueron previamente trasladados los ejes iníciales, muestra a la derecha del nuevo eje vertical, los BL globales superiores a la media, situación similar ocurriría con el FL, y sobre el eje horizontal se encuentran aquellas ramas que tiene un FL mayor que la media.

De los resultados obtenidos, que se muestran en el grafico 1, y adicionalmente se resumen en el anexo 2 , se observa cuál fue para el año de análisis la tipología de cada rama, por ejemplo, sectores claves en Magallanes resultaron ser: productos agrícolas (1); petróleo crudo y combustible y lubricante (6); vidrio, productos de vidrio, y productos de minerales no metálicos (20); servicios comerciales (28) y servicios de transporte ferroviario y caminero (30). Por su parte, también se observa, qué ramas son impulsoras de la economía, las que de acuerdo al criterio de Rasmussen, también se podrían considerar importantes (Rasmussen, 1956, pp. 135-136), a saber ellas son: frutas (2); pescados y mariscos frescos y productos del mar industrializados (5); otros minerales (8); aceites y grasas (10); productos químicos, de caucho y de plástico (19); muebles (23); otros productos manufacturados (24); gas y agua (26); servicios de hotelería y restaurantes (29); y servicios inmobiliarios y de propiedad de vivienda (36).

\section{ANÁLISIS ESTRUCTURAL DESDE LA PERSPECTIVA DE LA SENSIBILIDAD DE COEFICIENTES TÉCNICOS}

En lo que continua, su fundamento se sostiene en averiguar, ¿cuál es, la importancia que tiene cada una de las distintas etapas que forman las funciones de producción del sistema económico analizado?. Esto es, lo que sigue se centra en las elasticidades propias de cada actividad, es decir, lo que se abordará, guarda relación con los distintos efectos que tiene sobre la producción -caeteris páribus-, la alteración de algún coeficiente del sistema (o etapa productiva); para ello, se parte de la generación de unas alteraciones realizadas ex profeso, con el fin de cuantificar sus repercusiones sobre el sector en particular y el conjunto económico.

El planteamiento anterior, surge de los trabajos desarrollados en 1950 por Waugh y Sherman y Morrison, sin embargo, la idea fue aplicada a 
la economía, en concreto, a la matriz inversa de Leontief, por Evans en 1954, y es Sebald, en 1974, quien incorpora al procedimiento el concepto de "elasticidad", haciendo ver lo importante que es cuantificar los efectos que genera la alteración de un coeficiente técnico, sobre la inversa de Leontief y la producción total, $y$, a partir de estas ideas, en 1984 Schintke y, posteriormente, en 1988, Schintke y Stäglin, presentan una formulación qué serviría para identificar adecuadamente qué sector es más sensible, y para ello sostienen que un coeficiente será importante cuando una variación menor que el 100\%, es capaz de generar un cambio en la producción de alguna de las ramas en mayor medida que el nivel porcentual prefijado.

Sobre la base anterior, Schintke y Stäglin, señalan que, para el caso concreto de una variación del 1\%, la expresión 18 , cuantificará cuán sensible es $a_{i j}$ frente a dicha alteración. Luego $r_{i j}$ indicará cuál es el valor máximo, en porcentaje, que no provoca cambios superiores al 1\% en la producción de la i-ésima rama, o en otras palabras, señalará cuál es el porcentaje en que debe variar el coeficiente $a_{\mathrm{ij}}$ para que se produzca un cambio en la producción sectorial del 1 por ciento.

$$
r_{i j}(\%)=\frac{1}{a_{i j}\left[0.01 z_{i j}+z_{i j} \frac{x_{j}}{x_{i}}\right]}
$$

Donde $\mathrm{a}_{\mathrm{ij}} \in \mathrm{A} \mathrm{y}, \mathrm{z}_{\mathrm{ij}} \in \mathrm{Z}=(\mathrm{I}-\mathrm{A})^{-1}$.
De esta forma, una rama podrá considerase importante-caeteris páribus- de acuerdo al número de coeficientes $r_{i j}$ que presente, por ejemplo, menores que 15, tanto en columnas cómo en filas. Bajo este esquema, si una rama tiene muchos coeficientes importantes en columna, indicará cuán relevante son sus etapas productivas, para la demanda de output de otros sectores, por tanto, su importancia, se deberá asociar a las modificaciones tecnológicas del proceso de producción de las distintas ramas. Por su parte, un alto número de estos coeficientes en filas, será señal de la importancia que tienen los productos de esta actividad para el progreso de las otras, es decir, en este caso, su importancia se asociará a mejoras e innovaciones que se den en la producción (López y Pulido, 1993, pp. 176).

Posteriormente Hewings et al en 1989 y en Sonis y Hewing (1989; 1991 y 1992), se amplia el planteamiento anterior, evaluando la sensibilidad o variabilidad de pares de actividades económicas, introduciendo de esta forma el concepto de "campos de influencias". El enfoque de "campos de influencia" muestra el impacto de la alteración de un coeficiente $\mathrm{a}_{\mathrm{ij}}$ sobre otro $\mathrm{a}_{\mathrm{kl}}, \forall \mathrm{i} ; \mathrm{j}$, esto es, se toma un coeficiente $\mathrm{a}_{\mathrm{ij}}$, y se observa el efecto que produce su variación sobre el sistema. Entonces, si existe un elemento que destaque del resto y que sea importante para el coeficiente $\mathrm{a}_{\mathrm{ij}}$, pasarán a formar parte de lo que se denomina "campos de influencia de éste". Esta idea, fue aplicada posteriormente por Aroche en 1996; 2002 y 2005, utilizando para ello la formulación Schintke y Stäglin (1988) y Schnabl (1994). Su propuesta, consistió en hacer variar cada uno de los distintos coeficientes en forma separada, y observar las implicaciones que tal

Tabla 4: Actividades más importantes según relación de compra y venta entre ellas.

\begin{tabular}{|c|c|}
\hline \multicolumn{2}{|c|}{ Actividad que: } \\
\hline Compra & Vende \\
\hline construcción & vidrio y minerales no metálicos \\
\hline ganado; productos pecuarios y carne & ganado; productos pecuarios y carne \\
\hline servicios comerciales & transporte ferroviario y caminero \\
\hline petróleo (combustible y lubricante) & servicios empresariales \\
\hline petróleo (combustible y lubricante) & petróleo (combustible y lubricante) \\
\hline servicios empresariales & impresos y grabaciones \\
\hline petróleo (combustible y lubricante) & impresos y grabaciones \\
\hline construcción & hierro, acero y productos metálicos \\
\hline petróleo (combustible y lubricante) & transporte marítimo y aéreo \\
\hline ganado; productos pecuarios y carne & productos agrícolas \\
\hline
\end{tabular}

Fuente: Propia 
cambio tiene sobre la producción -caeteris páribus-, de esta forma, para una matriz de " $n$ "n" elementos, obtiene "n*n" matrices $y$, a partir de estas últimas, establece sus relaciones de dependencia por medio del uso de la teoría de grafos.

Una vez explicado lo anterior, lo que sigue será mostrar los principales resultados. Para ello se comienza por identificar las 10 actividades que presentan menores elasticidades $\left(r_{i j} \leq 5\right)^{16}$, es decir, por aquellas ramas que presentan "etapas productivas" que son más sensibles a los distintos cambios. Para estos efectos, se ha considerado que la variable $p$ tome el valor de un 1 por ciento ${ }^{17}$, resultados que se muestran en la tabla 4.

De acuerdo a lo expuesto, y la restricción impuesta -la cual, se debe recordar, es una limitación extrema-, se observa que las actividades más sensibles fueron las que se relacionan con la extracción de petróleo; servicios empresariales; construcción; transporte, y agricultura, en general. Sin embargo, ello no quiere decir, que tales ramas sean las más importantes bajo este esquema, esto simplemente indica, de momento, que ciertas etapas productivas son más sensibles que otras frente a posibles cambios.

En respuesta a lo anterior, es decir, a lo que guarda relación con la identificación de ramas más importantes, y fijando cómo limites para este caso un $r_{i j} \leq 20$, que es aún algo conservador, se observó que, en columnas, tales ramas fueron: frutas; pescados $y$ mariscos frescos y productos del mar industrializados; petróleo crudo y combustibles y lubricantes; otros mi-

16 Con el fin de facilitar la interpretación de los coeficientes $\mathbf{r}_{\mathbf{i j}}$, considere el siguiente ejemplo, si un coeficiente presenta un $\mathbf{r}_{\mathbf{i j}}=5$, éste se debe interpretar cómo: una variación del $5 \%$ en el coeficiente $\mathbf{a}_{\mathbf{i j}}$, con $\mathbf{a}_{\mathbf{i j}} \in \mathbf{A}$, pprovocaría un $1 \%$ de variación en la producción sectorial, claro está si se ha modificado todo en un 1\% (Pulido y Fontela, 1993, pp. 142).

17 Se ha elegido tal valor ya que autores como Pulido y Fontela (1993), Aroche (1996, 2002 y 2005), Ghosh y Roy (1998), Tarancón (2003) y Robles y Sanjuán (2005) consideran que el uso del 1\% es una medida habitual. Otros como Siebe (1996) se decantan por un 0.5\%, mientras que Bullard y Sebald (1977) lo hacen por el 2\%, y Jalili (2000) llega al extremo del 10\%. En este estudio, al querer ser más conservadores se opta por el $1 \%$. Se debe tener presente que si el valor elegido es bajo, se obtendrán pocos coeficientes importantes, esto es, se detectarán los más sensibles, y viceversa, cuando el valor considerado es más elevado, de igual modo, estos resultados fueron comparados con los que se obtiene de aplicar la técnica de West (1982), no encontrándose diferencias sustanciales entre ambos procedimientos.

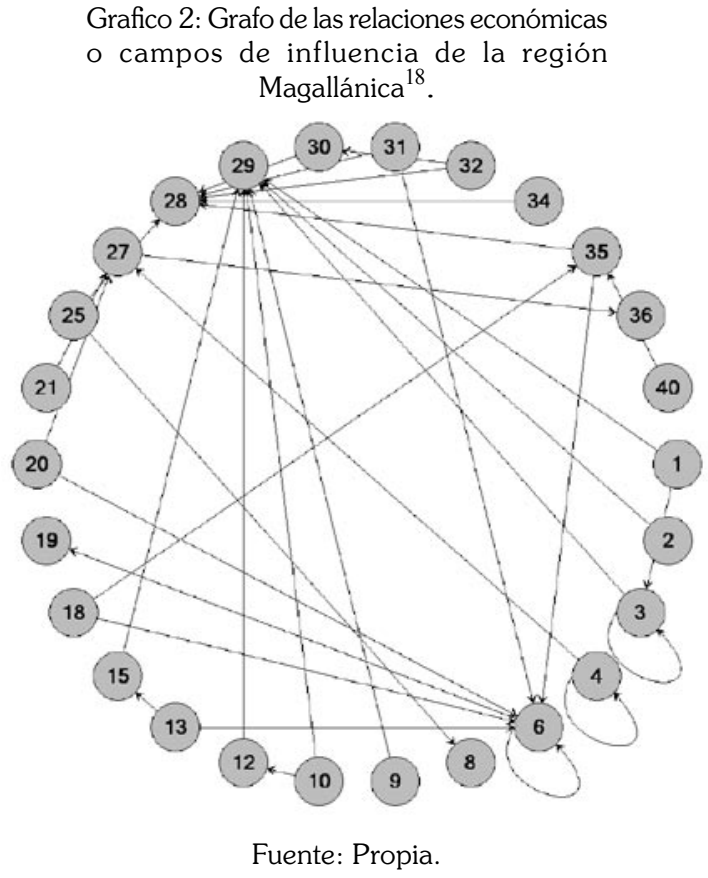

nerales; construcción; servicios comerciales; servicios de hotelería y de restaurantes, y servicios empresariales. De estos sectores, los que más coeficientes importantes presentan son: petróleo crudo y combustibles y lubricantes; construcción; servicios comerciales; servicios de hotelería y de restaurantes.

Por otra parte, cuando se repite el ejercicio anterior, pero ahora sólo considerando filas, se identificaron como ramas importantes, las siguientes: productos agrícolas; frutas; ganado; productos pecuarios y carne; productos silvícolas; madera y papel; petróleo crudo y combustible y lubricantes; otros minerales; aceites y grasas; otros productos alimenticios; impresos y grabaciones; vidrio, productos de vidrio, y productos de minerales no metálicos; electricidad; servicios de trasporte; comunicaciones; servicios empresariales; y de esparcimiento $y$ otros servicios. De estos, se pueden considerar como potenciales influyentes, por medio de su desarrollo tecnológico, para los distintos procesos de producción, a los siguientes sectores: productos silvícolas; transporte; servicios empresariales y, de esparcimiento y otros servicios.

18 En anexo 1 se puede ver cuál es la relación entre el número que aparece al interior del cada circulo y el sector que representa. 
Finalmente $y$, en referencia a un contexto global, esto es, de acuerdo al número de coeficientes más sensibles tanto en columnas cómo en filas, los sectores más importantes fueron; ganado; productos pecuarios y carne; petróleo crudo y combustible y lubricantes; otros minerales; construcción; servicios comerciales, hotelería y restaurantes; transporte en general; y servicios empresariales.

Con el fin de ampliar el análisis anterior, se procede a estudiar los campos de influencia de cada actividad en particular, ello con la intención de establecer cuál fue la relación de oferta y demanda entre las distintas etapas productivas. Sobre esta base, se considerará importante, a una rama, de acuerdo al número de coeficientes sensibles que presente, tanto en columnas cómo en filas. Para lograr este punto, se hace variar cada coeficiente por separado en un 1 por ciento $(p=1 \%)$, obteniéndose así un total de 1600 matrices y, a partir de esta perturbación, las matrices obtenidas y, luego de establecer cómo verdaderamente importante aquellos coeficientes que presenten un $\mathrm{r}_{\mathrm{ij}}$ menor o igual a 15 , se determinó por medio de la teoría de grafos, cuáles fueron las relaciones que tenía cada actividad con su entorno, es decir, se identificó cuál es el núcleo o la estructura económica de la región materia de estudio para el año considerado.

Realizar lo anterior, tiene un doble fin. Por un lado, permitirá saber cuán desarrollada estaba la economía analizada, ya que en la medida que más o menos entrelazado se encuentre el grafo resultante, será indicativo de cuan interrelacionados estaban sus actividades y, por otra parte, se sabrá cuáles eran los sectores articuladores de desarrollo, de acuerdo al número de flechas que llegan a él, así cómo las que salen del mismo.

Del ejercicio impuesto, el cual se resume en el gráfico 2, se puede constatar -esto es, para las condiciones establecidas; 40 ramas y un $r_{i j} \leq 15-$, que la región en cuestión estaba poco desarrollada, ya que presentó un alto número de ramas que, no sólo no se relacionaban con otras actividades, sino que además, las que lo hacían, estaban pocos interrelacionadas.

Por otro lado, se constata lo dependiente que era la economía de ciertas actividades, en concreto de 4: petróleo crudo, y combustible y lubricantes; construcción; servicios comerciales; y hotelería y restaurantes. Además, se observa que, otras ramas (tales como: agricultura; ganado; productos pecuarios y carne; productos silvícolas; maderas y papel; aceites y grasas; productos de molinería; pan, fideos y pastas; licores; cervezas; bebidas analcohólicas $y$ vinos; vidrio y minerales no metálicos; hierro, acero y productos metálicos; transporte y, servicios empresariales), presentaban cierta relación de dependencia, la que en comparación con la de los casos anteriores, es en menor grado, pero significativas respecto a un contexto global.

Del grafo resultante, también se desprende que la región estaba más bien vinculada a actividades ligadas al sector turismo, cómo se aprecia, existía una fuerte relación entre algunas ramas que son propias de este servicio, a saber; servicios comerciales; hotelería y restaurantes $y$, transportes varios $y$, no a otras tales como las derivadas del petróleo.

En este último sentido, se observa adicionalmente que la economía estaba sostenida, sobre lo que se podrían denominar 4 clúster. El primero estaría formado por los sectores: productos agrícolas con ganado (1); productos pecuarios y carne (3); productos silvícolas (4); productos de molinería; pan, fideos y pastas (12); servicios de hotelería y restaurantes (29), y servicios de transporte marítimo y aéreo (31). El segundo estaría compuesto por; petróleo crudo y combustible y lubricantes (6); productos alimenticios (13); impresos y grabaciones (18); servicios de transporte marítimo y aéreo (31), y servicios empresariales (35). Un tercer clúster se formaría por: vidrio y minerales no metálicos (20); hierro, acero y productos metálicos (21), y construcción (27). Finalmente el cuarto clúster lo compondrían los sectores; servicios comerciales (28), y servicios de trasporte ferroviario y caminero (30).

Del grafo obtenido, también se puede ver que fue el sector turismo el que aglutinó más actividades (6 ramas) en su entorno, esto es, fue una rama, que dependida del resto del sistema y este a su vez de él, por tanto, se constata nuevamente que la región en cuestión, se basaba más bien en esa actividad, y no en las ligadas a la minería, aún cuando esta última, se presentó como la que tenía las actividades más sensibles, sin embargo, no poseía un alto número de ellas, lo que hace que pierda relevancia respecto a otras. 


\section{LA REGIÓN DE MAGALLANES Y LA ANTÁRTICA CHILENA: BAJO UNA AMPLIA VISIÓN}

Finalmente, y con la idea de ampliar los resultados hasta ahora recogidos, esto es, aquellos referidos a la primera parte (encadenamientos), y los obtenidos a partir de la técnica de sensibilidad de coeficientes técnicos (elasticidades y campos de influencia), se procede a identificar las similitudes entre dichas respuestas, ello aún cuando se es consiente que estas técnicas no son comparables, ya que evalúan cosas completamente distintas. Sin embargo, se es de la idea que, a pesar de las diferencias que existen en la forma de abordar la importancia de una rama, su uso en conjunto es valido para estos fines, ya que lo verdaderamente relevante, en este caso, no es el procedimiento, sino las respuestas obtenidas.

Bajo la óptica anterior, se puede colegir que fueron importantes para el desarrollo económico de Magallanes las siguientes ramas: pescados y mariscos frescos y productos del mar industrializados (5); otros minerales (8); servicios comerciales (28); servicios de hotelería y restaurantes (29) y servicios empresariales (35). Y también ayudaron a ese proceso, pero en menor grado, los sectores: productos agrícolas (1); ganado; productos pecuarios y carne (3); petróleo crudo y combustibles y lubricantes (6); aceites y grasas (10); otros productos alimenticios (13); impresos y grabaciones (18); productos químicos, de caucho y de plástico (19); vidrio, productos de vidrio, y productos de minerales no metálicos (20); productos básicos de hierro y acero y productos metálicos (21); muebles (23); otros productos manufactureros (24); gas y agua (26); productos de la construcción (27); servicios de transporte (30 y 31); servicios de comunicaciones (33); servicios financieros y de seguros (34) y servicios inmobiliarios y de propiedad de vivienda (36).

\section{CONCLUSIONES}

El trabajo realizado consiste es un análisis ex- post de la economía magallánica basados en el enfoque input- output. En él se han identificado desde distintas ópticas de análisis económico, cuáles fueron las actividades más importantes para ella durante 1996, esto es, desde la perspectiva de enca- denamientos, sensibilidad de coeficientes técnicos y Campos de Influencia. Dichos resultados permitirían comprender de mejor forma las consecuencias de implementar determinadas políticas económicas, esto es, bajo el concepto de desarrollo de Albert Hirschman (1958), y considerando el supuesto caeteris páribus. Si se potencian las denominadas actividades importantes o claves, se esperaría un cierto nivel de desarrollo regional, aunque claro está, y la praxis así lo ha demostrado, la teoría dista mucho de la práctica, por ello tales consecuencias no se puede asegurar.

Se han calculado encadenamientos siguiendo las técnicas presentadas por Rasmussen (1956), Chenery y Watanabe (1958), Hazari (1970); Cella (1984), Sonis et al (1995), Dietzenbacher y van der Linden (1997) y Soza (2007). El hecho de contar con esta batería de indicadores, ha permitido obtener respuestas parecidas en general, aunque también disímiles. Por ello, se han calculado unos indicadores sintéticos que representan dicha información de la mejor manera posible.

Además, y en base al estudio de la sensibilidad de coeficientes técnicos, se han detectado en qué ramas existía una mayor elasticidad, se identificaron las ramas más importantes de acuerdo al número de coeficientes altamente sensibles que se presentan en columnas y filas, $y$, por último, una vez modificado por separado cada uno de los coeficientes técnicos, lo que significo procesar 1600 matrices de 40 ramas cada una, se construyó, por medio de la teoría de grafos, los campos de influencia de cada actividad.

Por otra parte, el trabajo aplicado ha servido para comprobar que la propuesta de Soza (2007), en la cual se consideran aspectos tales como un doble enfoque para la determinación de encadenamientos, la dispersión de compras y ventas, y la ponderación en base a las características de la economía, que entrega respuestas similares a las que se obtienen de aplicar las técnicas de Rasmussen (1956) y de Dietzenbacher y van der Linden (1997), propuestas que son generalmente utilizadas en el análisis, como medio de comparación. A modo de ejemplo, pueden verse los trabajos de Miller y Lahr (2001 y 2000), Panggaabean (2004), y Soza y Ramos (2003). Por tanto, se pueden aceptar como instrumentos de validación para esta propuesta. 
De igual forma, y en lo relativo a la identificación de ramas "claves", se observó que el planteamiento presentado por Soza, es coincidente con el enfoque de sensibilidad de coeficientes técnicos, en más casos que aquellas técnicas que le son precursoras (Rasmussen, Chenery- Watanabe, Hazari, Cella, Sonis et al y, Dietzenbacher- van der Linden). Esta situación se daría tanto para la identificación de ramas que son importantes, de acuerdo al número de coeficientes sensibles que se presentan en columnas y filas, así como para los campos de influencia que se forman. Esto es, aún cuando los tres enfoques, encadenamientos, sensibilidad de coeficientes técnicos y campos de influencia, abordan la problemática desde distintas ópticas y, por tanto, determinan los vínculos de una rama en forma "no comparable", ya que el proceso es distinto, ellas tienen en común la identificación de la importancia de una rama, es decir, lo relevante de estas ópticas es, y de allí la importancia de las similitudes en las respuestas, es la identificación de sectores motivadores de desarrollo, y no el proceso en cuestión, lo que hace que se acepten como validas ambas comparación.

Por otra parte, el trabajo realizado, ha permitido comprender los posibles resultados errados que se pueden obtener a la hora de identificar actividades importantes, cuando se aísla del análisis, e.g. el efecto tamaño que estas puedan presentar (ausencia de ponderación), así como, la distribución de los efectos de las compras y ventas del que depende una actividad (dispersión de las mismas sobre el entramado económico). Pues su ausencia, puede en el análisis tender a destacar una actividad como importante, no por el tipo de relaciones que ella posea, sino más bien, por su tamaño y lo concentrado de sus compras y ventas, situación que poco ayuda al desarrollo de una región.

Adicionalmente, se ha presentado, esto es, en términos de la estructura del trabajo realizado, una metodología de análisis estructural input- output, que proporciona una visión amplia de los distintos tipos de relación que tiene una rama, en un sentido de identificar la relevancia de ella por la importancia que tiene para el resto del sistema económico, es decir, por la vía de la cuantificación de su tira y empuje de actividades. De igual modo, dicha propuesta ha permitido identificar el impacto que tiene una alteración mínima de una etapa productiva, sobre el resto de la economía. Este hecho, ha permitido además identificar los campos de influencia de un sector, por una vía alternativa a la anterior, confirmándose por distintas vías, cuán importante puede resultar ser una rama.

Otro aspecto interesante de considerar, es que, el trabajo y las conclusiones, si bien es cierto, están referidas al año de la matriz y al supuesto caeteris páribus, los resultados obtenidos sin duda servirán para tomar decisiones de carácter político- económico con un mayor sustento, pues desde el punto de vista teórico y práctico, los respuestas logradas, y considerando la condición impuesta, debieran significar bajo el concepto de desarrollo o, de la importancia que tiene identificación de ramas motivadores de dinamismo desde la perspectiva de Rasmussen (1956), Hirschman (1958), Dietzenbacher y van der Linden (1997), Duarte et al (2002) o, Cai y Leung, en 2004 y 2005, entre otros, un pronto éxito en esta materia. Claro está que ello es difícil de asegurar, pues son muchas las variables involucradas, sin embargo, y bajo la perspectiva en que abordo esta temática, se cree que el ejercicio realizado ayuda a acercarse a tal objetivo, aunque claro está, no lo asegura.

Finalmente, y sobre la base de la forma en que se enfrentó el trabajo realizado, tiene la ventaja de facilitar a futuro, la comparación de los resultados obtenidos con los que se pueden derivar de las estructuras económicas de otras regiones que presentan ciertas condiciones de similitud respecto a la analizada, o bien, han alcanzado un determinado éxito en términos de desarrollo. De esta forma, dichas comparaciones pueden facilitar el plasmar decisiones que apunten a lograr cierto éxito en términos de desarrollo, aunque claro está, ellas no lo garantizaran. En este sentido, esta propuesta de análisis, se cree puede ayudar a potenciar a nivel local, las actividades que fueron exitosas para el año de referencia en otras regiones y que revisten cierto interés para la zona geográfica estudiada.

\section{AGRADECIMIENTOS}

El autor desea agradecer los comentarios y sugerencias vertidos por los tres árbitros anónimos y, por Flavia Morello y Marcelo Sasso (Universidad de Magallanes). Además desea agradecer en forma muy especial a Miguel Mora Cvitanic (Universidad de Ma- 
gallanes) por la ayuda que él presto en la elaboración del programa que permitió procesar las distintas matrices. Cualquier error u omisión es responsabilidad del infrascrito, no comprometiendo por tanto a la Revista MAGALLANIA, ni a la Universidad de Magallanes.

\section{BIBLIOGRAFÍA}

ANDREOSSO-O CALLAGHAN, Bernadette and YUE, Guoqiang. 2004. Intersectoral Linkages and Key Sectors in China 1987-1997. Asian Economic Journal, 18(2):165-183.

2000. Intersectoral Linkages and Key Sectors in China 19871997: An application of input-output linkage analysis. In: International input- output association, XIII International Conference on Input-Output Techniques, University of Macerata, Italy, August 21-25.

AROCHE, Fidel. 2005. Desisntegración en la Estructuta Productiva Mexicana y el Empleo. Los Coeficientes Importantes y su Importancia en la Integración. En: I Jornadas de Análisis Input-Output, Universidad de Oviedo, España, 22-23 de Septiembre.

2002. Structural Transformations and Important Coefficients in the North American Economies. Economic Systems Research, 14(3):257-273.

1996. Important Coefficients and Structural Change: A multi-layer approach. Economic Systems Research, $8(3): 235-247$

AUGUSTINOVICS, María. Methods of International and Intertemporal Comparison of Structure. In: CARTER Anne and Andrew BRÓDY, eds. Contributions to input-output analysis. Amsterdam, New York, Oxford, North-Holland Publishing Company, 1970. pp. 249-269.

BEYERS, Williams. 1976. Empirical Identification of Key Sectors: Some further evidence. Environment and Planning A, 8: 231-236.

BULLARD, Clark and SEBALD Anthony. 1977. Effects of Parametric Uncertainty and Technological Change on Input-Output Models. The Review of Economic and Statistics, 59(1):75-81.

CAI, Junning and LEUNG Pingsun. 2005. An Alternative Interpretation of the "Pure" Linkage Measures. The Annals of Regional Science, 39(1):39-54.

2004. Linkage Measures: a revisit and a suggested alternative. Economic Systems Research, 16(1):65-85

CASTRO, Marcos. Indicadores de Desarrollo Sostenible Urbano. Una aplicación para Andalucia. Tesis (Doctorado en Economía). Málaga, España, Universidad de Málaga, Departamento de Economía Aplicada, Estadística y Econometría, 2002, 540 pp.
CELLA, Guido. 1986. The Input-Output Measurement of Interindustry Linkages: A reply. Oxford Bulletin of Economics and Statistics, 48(4): 379-384.

1984. The Input-Output Measurement of Interindustry Linkages. Oxford Bulletin of Economics and Statistics, 46(1): 73-84.

CHENERY, Hollis and WATANABE, Tsunehiko. 1958. An International Comparison of the Structure of Production. Econométrica, 26(4): 487-521.

De CAEVEL, Jean, DEGUELDRE, Jean and PAELINCK, Jean. 1965. Analyse Quantitative de Certains Phénomènes du Développement Régional Polarisé, Essai de Simulation Statique D'itinéraires de Propagation. Collection de l'Institut de Science Economique de l'Université de Liège, Paris et Genève.

DIETZENBACHER, Erik and LINDEN, Jan van der. 1997. Sectoral and Spatial Linkages in the EC Production Structure. Journal of Regional Science, 37(2): 235-257.

DUARTE, Rosa, SÁNCHEZ-CHÓLIZ, Julio y BIELSA Jorge. 2002. Water Use in the Spanish Economy: An input-output approach. Ecological Economics, 43(1): 71-85

EVANS, Duane. 1954. The Effect of Structural Matrix Errors on Interindustry Relations Estimates. Econometrica, 22(4):461-480.

LÓPEZ, Ana María, y PULIDO, Antonio. 1993. Análisis de las Interrelaciones Sectoriales en España. Economía Industrial, 290: 167-178.

GHOSH, Ambica. A Note on Leontief Models with Non-Homogeneous Production Functions. En su: Planning programming and Input-output models: Selected papers on Indian planning. Monographs, University of Cambridge Department of Applied Economics at the University press, New York, 1968. p 45.

GHOSH, Santadas and ROY, Joyashree. 1998. Qualitative Input-Output Analysis of the Indian Economic Structure. Economic Systems Research, 10(3):263-272.

HAIR, Joseph, ANDERSON Rolph, TATHAM Ronald y William BLACK. Análisis Multivariante. Quinta edición, Madrid, Ed. Prentice Hall, 1999, 31-140 pp.

HAZARI, Bharat. 1970. Empirical Identification of Key Sectors in the Indian Economy. The Review of Economics and Statistics, 52(3):301-305.

HEWINGS, Geoffrey, FONSECA, Manuel and SONIS, Michael. 1989. Key Sectors and Structural Change in the Brazilian Economy: A comparison of alternative approaches and their policy implications. Journal of Policy Modeling, 11(1):67-90.

HIRSCHMAN, Albert. 1958. The Strategy of Economic Development. New Haven, Connecticut, USA, Yale University Press. 
JALILI, Ali Reza. 2000. Comparison of Two Methods of Identifying Input-Ouput Coefficients for Exogenous Estimation. Economic Systems Research, 12(1):113-129.

JONES, Leroy.1976. The Measurement of Hirschmanian Linkages. Quarterly Journal of Economics, 90(2): 323-333.

LEONTIEF, Wassily. Análisis Input-Output (1965). En su: Análisis Económico Input- Output. Segunda edición, España, Editorial Orbis, S. A., 1985. pp 226-227.

1951. The Structure of American Economy, 1919-1939. New York, Oxford University Press.

1946. Exports, Imports, Domestics Output and Employment. Quarterly Journal of Economics, 58(2):290-313.

1944. Output, Employment, Consumption and Investment. Quarterly Journal of Economics, 60(2):171-193.

1941. The Structure of American Economy, 1919-1929: An Empirical Application of Equilibrium Analysis. Harvard University Press.

1936. Quantitative Input and Output Relations in the Economic Systems of the United States. The Review of Economic Statistics, 18(3): 105-125.

LÓPEZ, Ana María, y PULIDO, Antonio. 1993. Análisis de las Interrelaciones Sectoriales en España. Economía Industrial, 290: 167-178.

MELLER, Patricio and MARFÁN, Manuel. 1981. Small and Large Industry: Employment generation, linkages and key sectors. Economic Development and Cultural Change, 29(2):263-274.

MILLER, Ronald. 1966. Interregional Feedback Effects in InputOutput Models: Some preliminary results. Papers of the Regional Science Association, 17:105-125.

MILLER, Ronald and LAHR, Michael. A Taxonomy of Extractions. In: Regional Science Perspectives in Economic Analizys (Ed) M.L. Lahr and R.M. Miller (North-Holland), March 2001, pp. 407-441.

2000. A Taxonomy of Extractions. In: International inputoutput association, XIII International Conference on Input-Output Techniques, University of Macerata, Italy, August 21-25.

PANGGABEAN, Martin. 2004. Regional Growth: Economically important sectors. Institute of Southeast Asian Studies, Singapore, ISEAS Working Paper, Visiting Researchers Series, 1: pp.1-24.

PÉREZ, César. Reducción de la Dimensión con Variables Cuantitativas: Componentes principales y análisis factorial. En su: Métodos Estadísticos Avanzados con SPSS. España. Thomson TM, 2005, pp. 489-532.

PULIDO, Antonio, y FONTELA, Emilio. 1993. Análisis InputOutput. Modelos Datos y Aplicaciones. España. Editorial Pirámide.
RAO, Vaman and Floyd HARMSTON. 1979. Identification of Key Sectors in a Region of a Developed Economy. Annals of Regional Science, 13(3): 78-90.

RASMUSSEN, Paul Noregaard. 1956. Studies in Inter-Sectoral Relations. Amterdam, North- Holland P. C.

ROBLES, Luís y SANJUÁN, Jesús. 2005. Key Sectors: Big Coefficients and Important Coefficients in Spain. In: I Jornadas de Análisis Input-Output, Universidad de Oviedo, España, 22-23 de Septiembre.

SCHINTKE, Joachim. 1984. Fehlersimulationen Mit Input-OuputTabellen des Statitischen Bundesamtes. Vierteljahrshefte zur Wirtschaftsforschung, 3:314-330.

SCHINTKE, Joachim and STÄGLIN Reiner. 1988. Important Input Coefficients in Market Transaction Tables and Production Flow Tables. In: CIASCHINI, M. (Ed), Input-Output Analysis, Chapman and Hall, New York, pp. 43-60.

SCHNABL, Hermann. 1994. The Evolution of Production Structures, Analyzed by a Multi-Layer Procedure. Economic Systems Research, 6(19):51-69.

SIEBE, Thomas. Important Intermediate Transactions and Multi-sectoral Modelling. 1996. Economic Systems Research, 8(2):183-194.

SEBALD, Anthony. 1974. An Analysis of the Sensitivity of Large Scale Input-Output Models to Parametric Uncertainties. Center for Advanced Computation, document no. 122, University of Illinois al Urbana.

SHERMAN, Jack and MORRISON, Winifred. 1950. Adjustment of and Inverse Matrix Correspondinding to a Change in One Element of a Given Matrix. The Annals of Mathematical Statistics, 21(1):124-127.

SONIS, Michael, GUILHOTO, Joaquim, HEWINGS, Geoffrey and MARTINS, Eduardo. 1995. Linkages, Key Sectors, and Structural Change: Some New Perspectives. The Developing Economics,33(3): 233-270.

SONIS, Michael and HEWINGS, Geoffrey. 1992. Coefficient Change in Input- Output Models: Thery and applications. Economic Systems Research. 4: 143-157.

1991. Fields of Influence and Extended Input- Output Analysis: A theorical account. In: J.H.Ll. Dewhurst, R.C. Jensen and G.J.D. Hewings (eds) Regional Input- Output Modeling - New Developments and Interpretations (Aldershot, Avebury), pp. 141-158.

1989. Error and sensitivity Input- Output Analysis: A new approach. In: R.E. Miller, K.R. Polenske and A.Z. Rose (eds) Frontiers of Input- Output Analysis (New York, Oxford University Press), pp. 232-244.

SOZA, Sergio. 2007. Análisis Estructural Input-Output: Antiguos problemas y nuevas soluciones. Tesis (Doctorado 
en Economía Aplicada). Oviedo, España, Universidad de Oviedo, Departamento de Economía Aplicada, 280 p.

2005. Análisis Estructural y su Comparación con los Métodos Clásicos de Análisis: Una aplicación empírica. En: TARANCÓN, Miguel Ángel y RAMOS, María del Carmen. Estructura Input-Output y Dinámica Económica. Editorial Club Universitario (ECU), España, 3: 35- 49.

2004. Análisis de la Economía Chilena a Partir de una Matriz Insumo-Producto, Economía y Administración. Universidad de Concepción, Concepción, Chile, 41(63): 63-86.

SOZA, Sergio y RAMOS, Carmen. 2005. Replanteamiento del análisis estructural a partir del análisis factorial. Una aplicación a economías europeas. Estudios de Economía Aplicada, 23(2): 363-384.

2003. Una doble perspectiva en el análisis de la estructura económica regional. Metodologías clásicas y de extrac- ción. En: Seminario Internacional sobre "Estadística y Desarrollo Local en un Mundo Globalizado y XXX Jornadas Nacionales de Estadística. Instituto de Estadística, Universidad Austral de Chile, InterAmerican Statistical Institute (IASI), Instituto Nacional de Estadística (INE Chile), 8-10 de Octubre, Valdivia, Chile.

STRASSERT, Günter. 1968. Zur Bestimmung Strategischer Sektoren Mit Hilfe von Input-Output Modellen. Jahrbücher für Nationalökonomie und Statistik, vol. 182(3): 211-215

TARANCÓN, Miguel Ángel. Técnicas de Análisis Económico Input-Output. Alicante, Editorial Club Universitario, 2003, 269p.

WAUGH, Frederick. 1950. Inversion of the Leontief Matriz by Power Series. Econometrica, 18(2):142-154. 
Anexo 1: Relación entre el número de sector y lo que representa.

\begin{tabular}{|c|c|}
\hline $\begin{array}{c}\text { Rama/ Sector } \\
\text { Número }\end{array}$ & Rama \\
\hline 1 & Productos Agrícolas \\
\hline 2 & Frutas \\
\hline 3 & Ganado; productos pecuarios y carne \\
\hline 4 & Productos silvícolas; Maderas y Papel \\
\hline 5 & Pescados y mariscos frescos y Productos del Mar Industrializados \\
\hline 6 & Petróleo crudo y combustibles y lubricantes \\
\hline 7 & Cobre \\
\hline 8 & Otros minerales \\
\hline 9 & Conservas de frutas y vegetales \\
\hline 10 & Aceites y grasas \\
\hline 11 & Productos lácteos \\
\hline 12 & Productos de molinería; Pan, fideos y pastas \\
\hline 13 & Otros productos alimenticios \\
\hline 14 & Alimentos para animales \\
\hline 15 & Licores; cervezas; bebidas analcohólicas y vinos \\
\hline 16 & Productos del tabaco \\
\hline 17 & Productos textiles, prendas de vestir, cuero y calzado \\
\hline 18 & Impresos y grabaciones \\
\hline 19 & Productos químicos, de caucho y de plástico \\
\hline 20 & Vidrio, productos de vidrio, y productos de minerales no metálicos \\
\hline 21 & Productos básicos de hierro y acero y productos metálicos \\
\hline 22 & Maquinaria y equipo eléctrico y no eléctrico \\
\hline 23 & Muebles \\
\hline 24 & Otros productos manufactureros \\
\hline 25 & Electricidad \\
\hline 26 & Gas y Agua \\
\hline 27 & Productos de la Construcción \\
\hline 28 & Servicios comerciales \\
\hline 29 & Servicios de hotelería y de restaurantes \\
\hline 30 & Servicios de transporte ferroviario y caminero \\
\hline 31 & Servicios de transporte marítimo y aéreo \\
\hline 32 & Servicios conexos de transporte \\
\hline 33 & Servicios de comunicaciones \\
\hline 34 & Servicios financieros y de seguros \\
\hline 35 & Servicios empresariales \\
\hline 36 & Servicios inmobiliarios y de propiedad de vivienda \\
\hline 37 & Servicios de administración pública \\
\hline 38 & Servicio de educación pública y privada \\
\hline 39 & Servicio de salud pública y privada \\
\hline 40 & Servicios de esparcimiento y otros servicios \\
\hline
\end{tabular}

Fuente: MIDELAN (00). 
Anexo 2: Tipo de encadenamiento e Importancia del sector según cantidad de Coeficientes Más Importantes (CMI) ${ }^{19}$.

\begin{tabular}{|c|c|c|c|c|c|c|c|c|c|}
\hline Rama & Rasmussen & Ch-W & Hazari & Cella & Sonis et al & D_vdL & Soza & \begin{tabular}{|l|} 
Factorial \\
\end{tabular} & CMI \\
\hline 1 & Clave & Clave & Isla & Clave & Clave & Clave & Isla & Clave & - \\
\hline 2 & Impulsor & Clave & Isla & Clave & Clave & Impulsor & Isla & Impulsor & - \\
\hline 3 & Clave & Clave & Clave & Isla & Isla & Impulsor & Isla & Isla & + \\
\hline 4 & Clave & Clave & Isla & Isla & Isla & Clave & Isla & Isla & - \\
\hline 5 & Impulsor & Impulsor & Clave & Impulsor & Isla & Impulsor & Impulsor & Impulsor & + \\
\hline 6 & Clave & Clave & Clave & Isla & Isla & Clave & Clave & Clave & ++ \\
\hline 7 & Isla & Isla & Isla & Isla & Isla & Isla & Isla & Isla & - \\
\hline 8 & Impulsor & Impulsor & Isla & Impulsor & Impulsor & Impulsor & Impulsor & Impulsor & + \\
\hline 9 & Isla & Isla & Isla & Impulsor & Impulsor & Isla & Isla & Isla & - \\
\hline 10 & Impulsor & Clave & Isla & Clave & Clave & Impulsor & Isla & Impulsor & - \\
\hline 11 & Isla & Isla & Isla & Isla & Isla & Isla & Isla & Isla & - \\
\hline 12 & Isla & Isla & Isla & Impulsor & \begin{tabular}{|l|} 
Isla \\
\end{tabular} & Isla & Isla & Isla & - \\
\hline 13 & Base & Base & Isla & Base & Base & Base & Isla & Base & - \\
\hline 14 & Isla & Isla & Isla & Isla & Isla & Isla & Isla & Isla & - \\
\hline 15 & Isla & Isla & Isla & Impulsor & Isla & Isla & Isla & Isla & - \\
\hline 16 & Isla & Isla & Isla & Isla & Isla & Isla & Isla & Isla & - \\
\hline 17 & Isla & Isla & Isla & Isla & Isla & Isla & Isla & Isla & - \\
\hline 18 & Base & Base & Isla & Base & Base & Base & Isla & Base & - \\
\hline 19 & Impulsor & Impulsor & Clave & Impulsor & Impulsor & Impulsor & Clave & Impulsor & - \\
\hline 20 & Clave & Clave & Isla & Base & Clave & Clave & Isla & Clave & - \\
\hline 21 & Base & Base & Isla & Base & Base & Base & Isla & Base & - \\
\hline 22 & Isla & Isla & Isla & Isla & Isla & Isla & Isla & Isla & - \\
\hline 23 & Impulsor & Impulsor & Isla & Impulsor & Impulsor & Impulsor & Isla & Impulsor & - \\
\hline 24 & Impulsor & Impulsor & Isla & Impulsor & Impulsor & Impulsor & Isla & Impulsor & - \\
\hline 25 & Clave & Clave & Isla & Base & Clave & Clave & Base & Base & - \\
\hline 26 & Impulsor & Impulsor & Isla & \begin{tabular}{|l|} 
Impulsor \\
\end{tabular} & \begin{tabular}{|l|} 
Impulsor \\
\end{tabular} & \begin{tabular}{|l|} 
Impulsor \\
\end{tabular} & Isla & \begin{tabular}{|l|} 
Impulsor \\
\end{tabular} & - \\
\hline 27 & Isla & Isla & Clave & Impulsor & Impulsor & Isla & Clave & Isla & + \\
\hline 28 & Clave & Impulsor & Clave & Impulsor & Impulsor & Impulsor & Clave & Clave & ++ \\
\hline 29 & Impulsor & Impulsor & Clave & Impulsor & Impulsor & Impulsor & Clave & Impulsor & ++ \\
\hline 30 & Clave & Clave & Isla & Base & Clave & Clave & Base & \begin{tabular}{|l|} 
Clave \\
\end{tabular} & - \\
\hline 31 & Clave & Clave & Isla & Base & Clave & Clave & Isla & Base & - \\
\hline 32 & Clave & Clave & Isla & Clave & Clave & Impulsor & Isla & Isla & - \\
\hline 33 & Clave & Clave & Isla & Base & Base & Clave & Isla & Base & - \\
\hline 34 & Base & Base & Isla & Base & Base & Base & Base & Base & - \\
\hline 35 & Base & Base & Isla & Base & Base & Base & Base & Base & + \\
\hline 36 & Isla & Impulsor & Clave & Impulsor & Impulsor & Isla & Clave & Impulsor & - \\
\hline 37 & Isla & Isla & Isla & Impulsor & Impulsor & Isla & Impulsor & Isla & - \\
\hline 38 & Isla & Isla & Isla & Isla & Isla & Isla & Isla & Isla & - \\
\hline 39 & Isla & Isla & Isla & Isla & Isla & Isla & Impulsor & Isla & - \\
\hline 40 & Clave & Clave & Isla & Base & Clave & Clave & Base & Base & - \\
\hline
\end{tabular}

Fuente: Propia.

19 Cuendo corresponde, las técnicas son corregidas por Ghosh; $\mathbf{C h}-\mathbf{W}=$ Chenery y Watanabe (1958); $\mathbf{D}$ _vdL= Dietzenbacher y van der Linden.; CMI = Coeficientes más importantes en columnas. El signo "-", se refiere a las ramas que presentan CMI en columnas bajo del promedio (tercer y cuarto cuartil), las con "+", a las que están sobre la media (segundo cuartil) y, con "++", sobre la media del promedio superior (primer cuartil). 